\title{
Solving Constrained Horn Clauses Modulo Algebraic Data Types and Recursive Functions
}

\author{
HARI GOVIND V K, University of Waterloo, Canada \\ SHARON SHOHAM, Tel-Aviv University, Israel \\ ARIE GURFINKEL, University of Waterloo, Canada
}

This work addresses the problem of verifying imperative programs that manipulate data structures, e.g., Rust programs. Data structures are usually modeled by Algebraic Data Types (ADTs) in verification conditions. Inductive invariants of such programs often require recursively defined functions (RDFs) to represent abstractions of data structures. From the logic perspective, this reduces to solving Constrained Horn Clauses (CHCs) modulo both ADT and RDF. The underlying logic with RDFs is undecidable. Thus, even verifying a candidate inductive invariant is undecidable. Similarly, IC3-based algorithms for solving CHCs lose their progress guarantee: they may not find counterexamples when the program is unsafe.

We propose a novel IC3-inspired algorithm RACER for solving CHCs modulo ADT and RDF (i.e., automatically synthesizing inductive invariants, as opposed to only verifying them as is done in deductive verification). RACER ensures progress despite the undecidability of the underlying theory, and is guaranteed to terminate with a counterexample for unsafe programs. It works with a general class of RDFs over ADTs called catamorphisms. The key idea is to represent catamorphisms as both $\mathrm{CHCs}$, via relationification, and RDFs, using novel abstractions. Encoding catamorphisms as CHCs allows learning inductive properties of catamorphisms, as well as preserving unsatisfiabilty of the original CHCs despite the use of RDF abstractions, whereas encoding catamorphisms as RDFs allows unfolding the recursive definition, and relying on it in solutions. Abstractions ensure that the underlying theory remains decidable. We implement our approach in Z3 and show that it works well in practice.

\section{CCS Concepts: • Theory of computation $\rightarrow$ Verification by model checking.}

Additional Key Words and Phrases: Formal verification, Algebraic Data Types, Recursive Functions, Model Checking

\section{ACM Reference Format:}

Hari Govind V K, Sharon Shoham, and Arie Gurfinkel. 2022. Solving Constrained Horn Clauses Modulo Algebraic Data Types and Recursive Functions. Proc. ACM Program. Lang. 6, POPL, Article 60 (January 2022), 29 pages. https://doi.org/10.1145/3498722

\section{INTRODUCTION}

Recursive data structures, such as Lists and Trees are ubiquitous in programming. Proofs of correctness of programs that use such data structures rely on the theory of Algebraic Data Types (ADT) - the logic counter-part to data structures, and on Recursively Defined Functions (RDF), to represent properties (or abstractions) of data types. For example, length of a List, height of a Tree, are captured by RDFs.

Authors' addresses: Hari Govind V K, Department of Electrical and Computer Engineering, University of Waterloo, Canada, hgvk94@gmail.com; Sharon Shoham, Tel-Aviv University, Israel, sharon.shoham@gmail.com; Arie Gurfinkel, Department of Electrical and Computer Engineering, University of Waterloo, Canada, arie.gurfinkel@uwaterloo.ca.

This work is licensed under a Creative Commons Attribution 4.0 International License.

(c) 2022 Copyright held by the owner/author(s).

2475-1421/2022/1-ART60

https://doi.org/10.1145/3498722

Proc. ACM Program. Lang., Vol. 6, No. POPL, Article 60. Publication date: January 2022. 


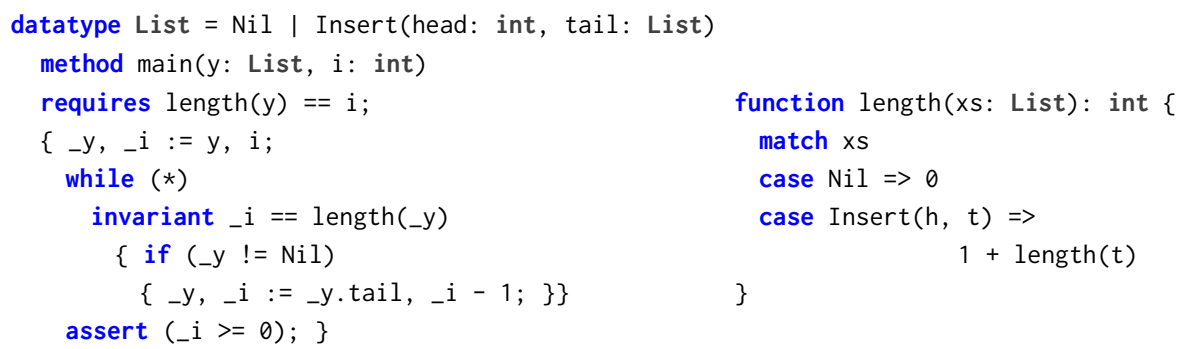

Fig. 1. A Dafny program that manipulates a list.

As an example, consider the imperative Dafny [Leino 2010] program in Fig. 1. The program manipulates a list represented by the variable y. Note that both the specification (in requires) and an inductive invariant (in invariant) require an RDF length (also shown in the figure). In this case, the RDF length appears both in the specification and the invariant, but that need not be the case. Similarly, programs without RDFs might require RDFs in their invariants (for example, via ghost code). Since the satisfiability of the combination of ADT and RDF is undecidable, even validating a candidate inductive invariant is difficult. While Dafny [Leino 2010], and similar deductive verification tools (e.g., Why3 [Filliâtre and Paskevich 2013], Viper [Müller et al. 2016]), provide ample support for reasoning with ADTs and RDFs, the situation is much worse in automated verification, where inductive invariants need to be synthesized and not just verified.

A prominent approach to automated verification, is to encode the verification condition of a given program as Constrained Horn Clauses (CHCs), such that the solutions to the CHCs correspond to the inductive invariants, and solve the $\mathrm{CHCs}$ using an off-the-shelf $\mathrm{CHC}$-solver. Several such solvers are available (e.g., [Champion et al. 2018; Fedyukovich et al. 2020; Hojjat and Rümmer 2018; Komuravelli et al. 2016]) and there is an annual competition [CHC-COMP 2021]. In the case of programs with data structures, such as the one in Fig. 1, the constraints of the CHCs are over a combination of theories including Linear Integer Arithmetic (LIA) (to model i), ADTs (to model $\mathrm{y}$ ), and RDFs (to model length). Supporting the combination of such theories in a CHC-solver is complicated because of the undecidability of even the most basic decision problems in this case.

In fact, several existing solvers, such as SPACER in Z3, already have some support for CHC modulo ADTs [Bjørner and Janota 2015]. However, as we show above, reasoning about ADTs almost always requires reasoning with RDFs as well, and RDFs are not supported. It is reasonable to view the relationship of RDFs to ADTs as the relationship between quantifiers and arrays - reasoning about array manipulating programs requires quantifiers for the same reasons as RDFs are needed for ADT-manipulating programs.

The key to our approach is a dual treatment of RDFs in CHCs $C$. On one hand, we approximate an axiom defining an $\mathrm{RDF}$ in $C$ by $\mathrm{CHCs}$ without RDFs, through a process called relationification, resulting in an equisatisfiable $\mathrm{CHC} C_{F}$. That is, $C_{F}$ preserves both proofs (satisfiability) and counterexamples (unsatisfiability). However, it does not preserve solutions - expressing a solution for $C_{F}$ might require a richer logic than the corresponding solution for $C$, since the former not only needs to capture an inductive invariant for the programs, but also needs to summarize the relationified $\mathrm{RDF}$. On the other hand, we approximate RDFs in $C$ by two novel abstractions that inject into $C$ a bounded unfolding of the RDF: both abstractions apply a finite unfolding of the recursive definition, after which the $k$-instantiation abstraction treats remaining RDF applications as uninterpreted functions, while the uninterpreted function abstraction omits them. In both abstractions, RDFs are replaced by their partial definition - thus, gaining decidability of basic queries. Relationification enables $\mathrm{CHC}$ solvers to deduce inductive summaries of RDFs whereas the abstractions provide 
unfoldings of RDFs. Inductive summaries and unfoldings of RDFs allow us to solve RDF constraints without facing the undecidability issues introduced by RDFs.

The combination of relationification with each of the abstractions ( $k$-instantiation or uninterpreted functions), parameterized by an unfolding bound $k$, results in two systems of abstract CHCs. Each system is an over-approximation (because of the abstraction). Nonetheless, relationification ensures that the abstract CHCs are equisatisfiable to the original ones. The $k$-instantiation abstraction is perfect for finding solutions, with the number of solutions preserved by the abstraction determined by the value of $k$. The uninterpreted function abstraction is a CHC over ADTs only and is perfect for showing unsatisfiability (i.e., finding counterexamples). The abstractions, parameterized by $k$, thus, create a hierarchy of abstractions (shown in Fig. 4).

We present an algorithm, RACER, that operates in the space of the hierarchy of $k$-instantiation abstractions, dynamically adjusting $k$, while simultaneously searching through the counterexamples in the uninterpreted function abstraction. While this sounds complex, it fits well into an IC3-style framework that iteratively explores bounded unfoldings of the system. Remarkably, this integration seamlessly combines learning inductive properties of RDFs with learning inductive invariants of the system, while exploiting the SMT support for non-inductive RDF reasoning via incremental unfolding of RDFs.

An important characteristic of our algorithm is that it is guaranteed to make progress and to discover counterexamples, if they exist. The problem of $\mathrm{CHC}$ solving is more general than SMTsolving (i.e., inferring invariants vs. checking invariants). Even CHC modulo LIA is undecidable, thus, there is little hope for completeness results when considering $\mathrm{CHC}$ solving. On the other hand, unsatisfiability of CHCs (and FOL, in general) is recursively enumerable. For CHCs, unsatisfiability corresponds to the existence of a counterexample to safety. As a result, progress - which implies, in particular, discovery of counterexamples - is key.

The integration of relationification with abstractions of RDFs is crucial for ensuring progress while preserving solutions of the CHCs. While $k$-deep unfolding alone does not suffice for making progress, relationification ensures progress as it allows to learn bounded properties of RDFs. On the other hand, relationification alone does not preserve solutions of the CHCs due to the inability to express summaries of RDFs. Thus, the combination of relationification with unfolding of RDFs is critical for maintaining progress while preserving (many) solutions. It allows to combine inference of inductive summaries of RDFs, which are needed to establish validity of solutions, with some unfoldings, guided by the property.

We develop a single procedure for solving CHCs with ADTs, RDFs, and LIA, while maintaining progress, and without affecting performance on LIA. This is in contrast to other related work, which either automates induction assuming that arithmetic is axiomatized [Reynolds and Kuncak 2015; Rosén and Smallbone 2015] or does not admit RDFs [Fedyukovich et al. 2020; Hojjat and Rümmer 2018; Komuravelli et al. 2016].

In our exposition, we restrict RDFs to catamorphisms (a.k.a. generalized folds). The RDF length in Fig. 1 is an example. Extending our ideas to other well defined RDFs is explained in Sec. 5.

Limitations. Our current approach is limited in two ways. First, we assume that the RDFs are specified. That is, we only synthesize inductive invariants, but not the supporting functions. Second, we do not synthesize new applications of RDFs, relying on a form of term abstraction [Alberti et al. 2012]. We leave addressing these orthogonal problems to future work.

Contributions. The paper makes the following contributions: (1) a new hierarchy of abstractions for $\mathrm{CHC}$ modulo ADT and RDF that combine relationification with bounded unfoldings of RDFs, thus, preserving counterexamples and (many) solutions; (2) an IC3-based procedure RACER for solving $\mathrm{CHC}$ modulo ADT and RDF that searches through the hierarchy of abstractions; It integrates 
into and extends the SPACER framework, and provides progress guarantees; (3) an implementation of RACER in Z3 that works with a combination of ADT, RDF, and other theories, such as LIA. (4) an empirical evaluation on a variety of benchmarks.

\section{OVERVIEW}

In this section we give an overview of our approach using a motivating example. Our focus is automatic safety verification of imperative programs that manipulate Algebraic Data Types (ADTs), such as lists, trees, or queues. Such programs and their specifications often require Recursively Defined Functions (RDFs), such as length, size, etc. Thus, we must support reasoning about the combination of ADTs and RDFs.

The key challenge of automated safety verification is automatically synthesizing and validating inductive invariants that summarize the behavior of all the loops. In case of ADTs, expressing invariants often requires RDFs, and, sometimes, RDFs that are not even present in the original program. For example, inductive invariants of list-manipulating programs often require to use the length of a list (and other recursive functions that compute inductive properties of lists). Thus, automatic reasoning over ADTs requires (a) synthesis of inductive invariants over ADTs and RDFs, and (b) synthesis of useful RDFs. In this paper, we focus on the first challenge - invariant synthesis. We assume that RDFs and their applications to program variables are either heuristically extracted from the program or are provided by the users. Synthesizing useful RDFs can be done as an abstraction-refinement loop over our work.

\subsection{Motivating Example}

To illustrate our approach, consider the list manipulating program in Fig. 1. List is a list ADT with two constructors $\mathrm{Nil}$ and Insert. length is an RDF that computes the length of a list. The method main takes as input a list $y$ and its length $i$. It removes elements from the list one by one in a loop, decreasing $i$ after each removal. Whenever the loop exits, it is asserted that the variable $i$ has a non negative value. The program is safe, as witnessed by the following inductive invariant $i==$ length $(y)$. While this program is simple, automatically verifying its safety is difficult for many existing verification techniques. Specifically, there are multiple theories involved that require to simultaneously reason about integer variables (i.e., variable $i$ ), ADT variables (i.e., variable y), and RDFs (i.e., function length). Furthermore, the proof that the suggested invariant is inductive requires guessing an inductive property of length: length $(y)$ is non-negative for any list $y$.

An effective technique for automatic program verification is reducing verification to satisfiability of Constrained Horn Clauses (CHCs). A CHC encoding of our example program from Fig. 1 is shown in Fig. 2a. In this encoding, the uninterpreted predicate Inv summarizes all behaviours of the main method. Essentially, it represents a desired inductive invariant of the loop. Inv takes three arguments: the first two are the same as those of main, and the third encodes the length of the list $y$. The third argument is required so that length(y) can appear in an inductive invariant.

A program is safe with respect to a specification if its $\mathrm{CHC}$ encoding is satisfiable (as a First Order Logic formula). A solution to CHCs is a mapping from all of the uninterpreted predicates to FOL formulas (in some theory), such that replacing predicates by the corresponding formulas makes the resulting FOL formula valid. For instance, in the $\mathrm{CHC}$ encoding of our example (Fig. 2a), a solution assigns to predicate $\operatorname{Inv}(y, i, j)$, the formula $(i=j)$, where $j$ encodes the length of list $\mathrm{y}$, as captured by the constraint length $(y)=j$. Hence, this is essentially the inductive invariant $i==$ length $(y)$ Representing the RDF application length $(y)$ by $j$ follows the idea of term abstraction. It allows us to separate the task of synthesizing the required RDF applications from the task of inferring inductive invariants over them, which is the focus of the paper. Namely, instead of synthesizing RDF applications in the process of inferring an invariant (solution), we infer invariants over variables 


$$
\begin{aligned}
\operatorname{length}(y)=i & \Rightarrow \operatorname{Inv}(y, i, i) \\
\operatorname{Inv}(y, i, j) \wedge \operatorname{length}(y)=j \wedge y \neq \operatorname{nil} \wedge y^{\prime}=\operatorname{tail}(y) \wedge \operatorname{length}\left(y^{\prime}\right)=j^{\prime} & \Rightarrow \operatorname{Inv}\left(y^{\prime}, i-1, j^{\prime}\right) \\
\operatorname{Inv}(y, i, j) \wedge \operatorname{length}(y)=j \wedge i<0 & \Rightarrow \perp
\end{aligned}
$$

(a) $\mathrm{CHC}$ modulo ADT and RDF encoding safety of the program from Fig. 1.

$$
\begin{aligned}
y=\operatorname{nil} \wedge z=0 & \Rightarrow \operatorname{Length}(y, z) \\
y \neq \operatorname{nil} \wedge \operatorname{Length}(\operatorname{tail}(y), l) \wedge z=1+l & \Rightarrow \operatorname{Length}(y, z) \\
\operatorname{Length}(y, i) & \Rightarrow \operatorname{Inv}(y, i, i) \\
\operatorname{Inv}(y, i, j) \wedge \operatorname{Length}(y, j) \wedge y \neq \operatorname{nil} \wedge y^{\prime}=\operatorname{tail}(y) \wedge \operatorname{Length}\left(y^{\prime}, j^{\prime}\right) & \Rightarrow \operatorname{Inv}\left(y^{\prime}, i-1, j^{\prime}\right) \\
\operatorname{Inv}(y, i, j) \wedge \operatorname{Length}(y, j) \wedge i<0 & \Rightarrow \perp
\end{aligned}
$$

(b) $\mathrm{CHCs} C_{F}$ obtained by relationifying the RDFs in Fig. 2a

$$
\begin{aligned}
y=\operatorname{nil} \wedge z=0 & \Rightarrow \operatorname{Length}(y, z) \\
y \neq \operatorname{nil} \wedge \operatorname{Length}(\operatorname{tail}(y), l) \wedge z=1+l & \Rightarrow \operatorname{Length}(y, z) \\
\operatorname{Length}(y, i) \wedge i=\operatorname{length}(y) & \Rightarrow \operatorname{Inv}(y, i, i) \\
\operatorname{Length}(y, j) \wedge j=\operatorname{length}(y) \wedge \operatorname{Length}\left(y^{\prime}, j^{\prime}\right) \wedge j^{\prime}=\operatorname{length}\left(y^{\prime}\right) \wedge & \\
\operatorname{Inv}(y, i, j) \wedge y \neq \operatorname{nil} \wedge y^{\prime}=\operatorname{tail}(y) & \Rightarrow \operatorname{Inv}\left(y^{\prime}, i-1, j^{\prime}\right) \\
\operatorname{Length}(y, j) \wedge j=\operatorname{length}(y) \wedge \operatorname{Inv}(y, i, j) \wedge i<0 & \Rightarrow \perp
\end{aligned}
$$

(c) $C_{f F}$ : the result of adding back the length RDF to relationified $\mathrm{CHCs} C_{F}$ from Fig. 2b.

Fig. 2. Example CHCs for (a) encoding of Dafny program from Fig. 1; (b) relationification, (c) relationifications with RDFs. Modifications and additions are highlighted in yellow and green, respectively.

that represent a predefined set of RDF applications. With this encoding, deciding whether the original program is safe is reduced to inferring solutions to the corresponding $\mathrm{CHC}$ encoding of its verification condition. We stress that inferring solutions (i.e., inductive invariants) is much harder than checking that a candidate invariant is inductive, as traditionally done in deductive verification.

Note that it is possible that a set of CHCs is satisfiable, but has no solution in a given theory. That is, the FOL model that witnesses satisfiability is not definable in the fixed language of solutions. On the flip side, a program is unsafe with respect to a specification if its $\mathrm{CHC}$ encoding is unsatisfiable. In this case, the program exhibits a (finite) counterexample: a feasible execution of the program that violates the specification. From perspective of $\mathrm{CHCs}$, this means that there is a refutation resolution proof (i.e., a proof deriving false) from the $\mathrm{CHCs}$.

\subsection{Challenges for Solving CHCs Modulo RDFs}

Solving CHCs is a challenging problem in general, and has received a lot of attention (e.g., [De Angelis et al. 2020; Fedyukovich et al. 2017; Grebenshchikov et al. 2012; Hoder and Bjørner 2012; Hojjat and Rümmer 2018; Komuravelli et al. 2016; Zhu et al. 2018]). CHCs modulo ADTs and RDFs bare additional challenges: 


$$
\begin{aligned}
& y=\operatorname{nil} \wedge z=0 \Rightarrow \operatorname{Length}(y, z) \\
& y \neq \operatorname{nil} \wedge \operatorname{Length}(\operatorname{tail}(y), l) \wedge z=1+l \Rightarrow \operatorname{Length}(y, z) \\
& \operatorname{Length}(y, i) \wedge i=\operatorname{length}_{u f}(y) \wedge i=i t e\left(y=n i l, 0,1+\operatorname{length}_{u f}(\operatorname{tail}(y))\right) \Rightarrow \operatorname{Inv}(y, i, i)
\end{aligned}
$$

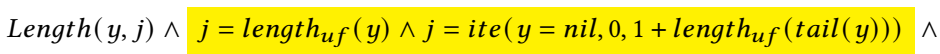

$$
\begin{aligned}
& \operatorname{Length}\left(y^{\prime}, j^{\prime}\right) \wedge j^{\prime}=\text { length }_{u f}\left(y^{\prime}\right) \wedge j^{\prime}=\operatorname{ite}\left(y^{\prime}=\operatorname{nil}, 0,1+\operatorname{length}_{u f}\left(\operatorname{tail}\left(y^{\prime}\right)\right)\right) \wedge \\
& \operatorname{Inv}(y, i, j) \wedge y \neq \operatorname{nil} \wedge y^{\prime}=\operatorname{tail}(y) \Rightarrow \operatorname{Inv}\left(y^{\prime}, i-1, j^{\prime}\right) \\
& \operatorname{Length}(y, j) \wedge j=\operatorname{length}_{u f}(y) \wedge j=i t e\left(y=n i l, 0,1+\operatorname{length}_{u f}(\operatorname{tail}(y))\right) \wedge \\
& \operatorname{Inv}(y, i, j) \wedge i<0 \Rightarrow \perp
\end{aligned}
$$

(a) $C_{f F}^{1}$ : the 1-instantiation abstraction of $\mathrm{CHCs} C_{f F}$ from Fig. 2c. length $h_{u f}$ is an uninterpreted function.

$$
\begin{aligned}
& y=\text { nil } \wedge z=0 \Rightarrow \text { Length }(y, z) \\
& y \neq \operatorname{nil} \wedge \operatorname{Length}(\operatorname{tail}(y), l) \wedge z=1+l \Rightarrow \operatorname{Length}(y, z) \\
& \text { Length }(y, i) \wedge i=i t e(y=n i l, 0,1+w) \Rightarrow \operatorname{Inv}(y, i, i) \\
& \operatorname{Length}(y, j) \wedge j=\operatorname{ite}(y=n i l, 0,1+w) \wedge \operatorname{Length}\left(y^{\prime}, j^{\prime}\right) \wedge j^{\prime}=\operatorname{ite}\left(y^{\prime}=\operatorname{nil}, 0,1+w^{\prime}\right) \wedge \\
& \operatorname{Inv}(y, i, j) \wedge y \neq \operatorname{nil} \wedge y^{\prime}=\operatorname{tail}(y) \Rightarrow \operatorname{Inv}\left(y^{\prime}, i-1, j^{\prime}\right) \\
& \operatorname{Length}(y, j) \wedge j=\operatorname{ite}(y=n i l, 0,1+w) \wedge \operatorname{Inv}(y, i, j) \wedge i<0 \Rightarrow \perp
\end{aligned}
$$

(b) $C_{f F}^{k \uparrow}$ : Uninterpreted functions abstraction of $\mathrm{CHCs} C_{f F}$ from Fig. 2c.

Fig. 3. Examples of (a) $k$-instantiation and (b) uninterpreted function abstractions of CHCs from Fig. 2c.

Undecidability of validating solutions. In the presence of RDFs, determining validity of FOL formulas is undecidable. Thus, even checking whether a candidate formula is an inductive invariant (a step used by many existing verification techniques) is undecidable. Validity may depend on properties of RDFs that must be established inductively (i.e., no amount of finite unfolding of the recursive definition is sufficient). Applying induction requires coming up with an inductive hypothesis, which, intuitively, is a source of undecidablity.

The undecidability challenge significantly limits applicability of guess-and-check techniques, such as Houdini [Flanagan et al. 2001; Flanagan and Leino 2001], in this context. This extends to all guess-and-check techniques including more recent approaches based on machine learning [Garg et al. 2016] and grammar-based synthesis [Fedyukovich et al. 2020]. One exception is the CVC4 SMT solver that combines candidate enumeration with induction [Reynolds and Kuncak 2015]. However, the technique in [Reynolds and Kuncak 2015] is not fully automated (but rather user or problem guided). It assumes that required inductive hypotheses are either part of the input, or are provided by the user as sub-goals. This is justified by their application domain (automating deductive verification), but is not very effective in an automated verification setting, as recently shown in [De Angelis et al. 2020]. There are also known sub-classes of RDFs for which validity is decidable. One such class of infinitely surjective catamorphisms (ISCs) over ADTs is presented by Suter et al. [Suter et al. 2010]. However, it is not clear that determining that an RDF is of an appropriate class (i.e., an ISC) is decidable.

Incompleteness of RDF elimination. As RDFs make validating solutions undecidable, a natural direction of attack is to reduce the problem by somehow eliminating RDFs, while preserving the key characteristics of the verification task. One approach is relationification: encoding RDFs as 
$\mathrm{CHC}$ predicates, constrained by additional Horn clauses. For example, relationification of the $\mathrm{CHCs}$ in Fig. 2a gives us the CHCs in Fig. 2b. Relationified CHCs do not contain RDFs. Instead, they are over ADTs (and other background theories). Therefore, checking the validity of solutions is decidable. Intuitively, relationification replaces recursive functions by their implementation. One of our results is to show that while relationification preserves satisfiability, it loses solutions over the language of CHCs. Roughly, this is because a solution to the CHCs should now also include a summary of the relationified RDF. However, there are cases where such a summary cannot be specified without RDFs (see Ex. 5.8).

\subsection{Our Approach}

In this paper, we present a new approach for solving CHCs modulo ADT and RDF that addresses the above challenges. The core of our approach is the combination of relationified predicates with a novel abstraction of RDFs based on finite RDF unfoldings.

The use of (abstraction of) RDFs allows to express solutions that cannot be expressed without RDFs, thus, tackling the second challenge. Abstractions are key to ensuring that checking validity of solutions remains decidable (tackling the first challenge). Unfortunately, abstractions may not be sufficient to validate solutions, specifically, in the cases where finite unfoldings do not suffice. This is where the interplay with the relationified predicates, that are conjoined with the RDF abstractions, comes into play: relationified predicates allow to use Property Directed Reachability (PDR) to automatically generate necessary inductive hypotheses and lemmas that are needed in order to validate solutions. However, this is not the only role of the relationified predicates. The Horn clauses that encode the relationified predicates ensure that despite the RDF abstraction, unsatisfiability is preserved. Namely, if the original CHC is unsatisfiable, so is the transformed one. Indeed, a key characteristic of our approach is that it is refutationally complete - whenever the (original) $\mathrm{CHCs}$ are unsatisfiable (i.e., the program is unsafe), our approach is guaranteed to terminate with a finite counterexample. In fact, our approach has a stronger property from which the former follows: the approach is ensured to make progress in verifying bounded safety for increasing bounds.

Relationification side by side with RDFs. The first step in our approach is to extend a given set of $\mathrm{CHCs}$ modulo RDFs with relationification of the RDFs. That is, we conjoin the CHCs with clauses that constrain the relationified predicates and conjoin each RDF application with an application of the relationified predicate. For example, the result of this transformation on CHCs in Fig. 2a is shown in Fig. 2c. The newly added clauses, and newly added predicate applications are highlighted in green and yellow, respectively. The resulting $\mathrm{CHCs}$ preserve both solutions of the original $\mathrm{CHCs}$ (when they are satisfiabile), and unsatisfiability (when the original CHCs are unsatisfiable).

Abstractions of RDFs. The second step in our approach is a new IC3-style algorithm, called RACER, that employs two orthogonal abstractions simultaneously in order to solve the augmented CHCs. These abstractions, called $k$-instantiation and uninterpreted functions abstraction, respectively, are key to avoiding the undecidability barrier (for solution validation).

The $k$-instantiation abstraction unfolds all occurrences of RDFs in a formula $k$ times and replaces any remaining RDFs with uninterpreted functions (UF). Applying the $k$-instantiation abstraction to CHCs modulo ADTs and RDFs, gives us a set of abstract CHCs modulo ADTs and UF. ${ }^{1}$ The result of applying the 1-instantiation abstraction to the CHCs in Fig. 2c is shown in Fig. 3a. We show that, similar to the other transformations, the $k$-instantiation abstraction of $\mathrm{CHCs}$ is equi-satisfiable to the original CHCs, but, more importantly, it has more solutions than relationified CHCs. In fact, there is a hierarchy: all solutions to $(k-1)$-instantiation abstraction are also solutions to

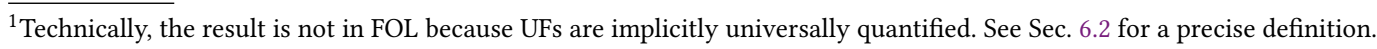


$k$-instantiation abstraction (Fig. 4), and all of them are solutions to the original CHCs. Furthermore, validating solutions of the abstraction of the CHCs is decidable, as it only involves ADTs and UF, but no RDFs. Hence, the $k$-instantiation abstraction is particularly useful for finding solutions. Note that while the abstraction makes validating solutions decidable, relationified predicates provide the supporting inductive lemmas (about RDFs) that are often necessary for solutions to be validated.

The uninterpreted function abstraction removes all occurrences of UF from a given formula (a detailed definition is given in Sec. 6.1). Thus, it results in even simpler CHCs - without UFs. An example of the uninterpreted functions abstraction of the CHCs in Fig. 3a is shown in Fig. 3b. The uninterpreted function abstraction also preserves (un)satisfiability, thanks to the relationified predicates. This abstraction is useful for proving unsatisfiability of the original $\mathrm{CHCs}$, as refutational completeness of first order logic ensures the existence of a (finite) refutation proof for the abstraction.

While the description above might suggest that we explicitly construct the abstractions, this is not the case. The abstractions are tightly integrated as part of an IC3-style algorithm, RACER ( Alg. 1 in Sec. 7). RACER uses $k$-instantiation abstraction to block infeasible counterexamples and to infer useful inductive lemmas over the predicates (including the predicates that define RDFs), and uninterpreted function abstraction to extend partial counterexamples (until they are either shown to be infeasible or become complete). The value for $k$, in both abstractions, is adjusted dynamically by RACER. While RACER does not guarantee to terminate - the underlying problem, after all, is undecidable, it does guarantee to find a counterexample if one exists.

For our running example, RACER produces the following solution:

$$
\operatorname{Inv}(y, i, j) \triangleq i=j \quad \operatorname{Length}(y, j) \triangleq j \geq 0
$$

Note that the solution for Length is an inductive property of the length RDF.

Outline. The rest of the paper is structured as follows. We review the necessary background in Sec. 3, and define CHC modulo ADTs and RDFs in Sec. 4. Reduction from CHC modulo RDF to $\mathrm{CHC}$ is shown in Sec. 5, followed by the definition of RDF abstractions for CHCs in Sec. 6. The main algorithm is presented in Sec. 7 and evaluated in Sec. 8. Related work is discussed in Sec. 9 and Sec. 9 concludes the paper.

\section{BACKGROUND}

In this section, we provide a brief background on first order logic, satisfiability modulo theories, the theory of ADTs, which this paper focuses on, and Constrained Horn Clauses.

\subsection{Satisfiability Modulo Theories}

We work in standard many-sorted First Order Logic with equality. Terms and formulas are defined over a signature $\Sigma$ which consists of a set of sorts, a set of predicate symbols and a set of function symbols, each with a designated non-negative arity and sorts. We refer to zero-ary function symbols as constants. A term $t$ is either a (sorted) variable $x$, or a function symbol applied to terms, $f\left(t_{1}, \ldots, t_{n}\right)$, respecting the arity and sorts of $f$. An atomic formula is an equality applied to terms of the same sort, $t_{1}=t_{2}$, or a predicate symbol applied to terms, $p\left(t_{1}, \ldots, t_{n}\right)$, respecting the arity and sorts of $p$. A formula is a Boolean combination of atomic formulas, possibly with quantification. We use the special term ite $\left(c, t_{1}, t_{2}\right)$, where $c$ is a formula, and $t_{1}, t_{2}$ are terms of the same sort, to denote an "if then else" operation. A model $M$ consists of a nonempty domain for each sort, and a valuation function that maps each function and predicate symbol to its interpretation. For a closed formula $\varphi, M \vDash \varphi$ is defined in the usual way.

We assume an underlying theory $\mathcal{T}$ that interprets some sorts, function and predicate symbols. A symbol interpreted by the theory is called an interpreted symbol. We refer to interpreted constants 
as values. Theory $\mathcal{T}$ may either be defined by a set of axioms (in which case the theory consists of the deductive closure of the axioms), or by a set of intended models (in which case the theory consists of all formulas that are true in these models). We consider satisfiability modulo theories, and say that a formula $\varphi$ is satisfiable modulo theory $\mathcal{T}$ if there is a model $M$ of $\mathcal{T}$ that satisfies the formula, denoted $M \models_{\mathcal{T}} \varphi$. Otherwise, the formula is unsatisfiable. $\varphi$ is valid modulo $\mathcal{T}$ if every model of $\mathcal{T}$ satisfies $\varphi$. We say that two formulas, $\theta_{1}$ and $\theta_{2}$, are equisatisfiable, denoted $\theta_{1} \approx \theta_{2}$, if it holds that there exists $M \models_{\mathcal{T}} \theta_{1}$ if and only if there exists $M^{\prime} \vDash_{\mathcal{T}} \theta_{2}$. We omit $\mathcal{T}$ and write $M \vDash \varphi$ when the theory is clear from context.

By convention, we use non bold symbols (like $f$ ) to refer to syntactic entities and bold symbols (like $f$ ) to refer to their semantics i.e., their valuation in a model. Given a model $M$ over a signature $\Sigma$ that may or may not include $a$, we use the notation $M[a \mapsto \boldsymbol{a}]$ to mean the model over $\Sigma \cup\{a\}$ obtained from $M$ when $a$ is interpreted to $\boldsymbol{a}$.

Literals and clauses are defined as usual: a literal is an atomic formula or its negation, a clause is a disjunction of literals. Throughout the paper, we say that a formula $\theta$ is pure with respect to a function symbol $f$, if $f$ only appears in positive literals of the form $f(\bar{t})=x$ where $f$ does not appear in $\bar{t}$, and $x$ is a variable. We call such formulas $f$-pure to emphasize $f$. Note that every quantifier-free formula $\theta$ can be converted into an equivalent $f$-pure formula by introducing existentially quantified variables that represent the $f$ applications. For example, $p(f(f(x)))$ may be rewritten into $\exists y, z \cdot f(x)=y \wedge f(y)=z \wedge p(z)$.

\subsection{Theory of Algebraic Data Types}

The theory of Algebraic Data Types (ADT) is defined over a signature $\Sigma$ containing a sort $\tau$ for an algebraic datatype (for simplicity, we assume only one such sort). The signature $\Sigma$ includes function symbols for constructors and selectors, and predicate symbols for testers. A constructor for sort $\tau$, denoted $C$, returns an element of sort $\tau$ (its arguments may be of any sort, including $\tau$ ). A selector for the $i$ th argument of $C$, denoted $S_{C}^{i}$, receives an element of sort $\tau$ and returns an element of the sort of the $i$ th argument of $C$. A tester for $C$, denoted $I S_{C}$, is defined over sort $\tau$. The theory of ADTs is defined by an initial model of the following axiom schema, $\xi$ :

$$
\forall \bar{x} \cdot I S_{C}(C(\bar{x})) \quad \forall \bar{x} \cdot \neg I S_{C^{\prime}}(C(\bar{x})) \quad \forall \bar{x} \cdot S_{C}^{i}(C(\bar{x}))=x_{i}
$$

where $\bar{x}=\left\{x_{1}, \ldots, x_{n}\right\}$ and $C \neq C^{\prime}$. An initial model is a model where the domain of sort $\tau$ consists of all constructor terms: these are ground terms where the only ADT function applications are of constructors. The theory of ADT consists of the set of formulas that hold in an initial model of $\xi$. The theory of ADTs is decidable (even with quantifiers) [Zhang et al. 2004].

Note that an initial model cannot be specified in First Order Logic. However, many SMT solvers support reasoning over it. Accordingly, in the sequel, all models we consider are isomorphic to an initial model. In particular, we write $M \models \theta$ to mean that $M$ is isomorphic to an initial model that satisfies $\theta$.

The List ADT. For convenience of presentation, throughout the paper, we use a combination of the List ADT and Linear Integer Arithmetic, i.e., Lists of Integers, denoting them simply as Lists. We stress that our approach is more general. It applies to other ADTs, including mutually recursive ones. The List datatype is defined using a signature that consists of sort $\mathrm{s}$ for the elements stored in lists, which is in our case sort Int, and sort List ${ }_{\mathrm{s}}$ for lists. It has two constructors:

- nil is a nullary constructor for constructing an empty list, and

- insert receives an element of sort $\mathrm{s}$ and a list and constructs a list where the element is added to the head of the list. 
The insert constructor is associated with two selectors: head for the first argument, and tail for the second argument.

\subsection{Constrained Horn Clauses}

Given a signature $\Sigma$, a background theory $\mathcal{T}$ over $\Sigma$, and a set $P$ of uninterpreted predicate symbols not in $\Sigma$ (but defined over the sorts in $\Sigma$ ), a Constrained Horn Clause (CHC) is a closed formula of the form

$$
\forall \bar{x}, \bar{x}^{\prime} \cdot \phi\left(\bar{x}, \bar{x}^{\prime}\right) \wedge \bigwedge_{i} p_{i}\left(\bar{x}_{p_{i}}\right) \Rightarrow h\left(\bar{x}_{h}^{\prime}\right)
$$

where $p_{i} \in P, h \in P \cup\{\perp\}, \bar{x}^{\prime}=\left\{x^{\prime} \mid x \in \bar{x}\right\}, \phi$ is a quantifier-free formula over $\sum$ with free variables in $\bar{x} \cup \bar{x}^{\prime}, \bar{x}_{p_{i}} \subseteq \bar{x}$, and $\bar{x}_{h}^{\prime} \subseteq \bar{x}^{\prime}$. The same unknown predicate $p \in P$ can appear multiple times in a $\mathrm{CHC}$ with different arguments (i.e., in the equation above $p_{i}$ is not necessary distinct from $p_{j}$ when $i \neq j$ ). The left hand side of the implication is called the body or tail of the CHC, and the right hand side is called its head. We refer to $\phi$ as the constraint. A set of CHCs is a conjunction of CHCs. With abuse of notation, we sometimes refer to a set of $\mathrm{CHCs}$ as a $\mathrm{CHC}$. A (single) $\mathrm{CHC}$ is linear if the tail has at most one uninterpreted predicate symbol. A set of CHCs is linear if all the $\mathrm{CHCs}$ in it are linear. Otherwise, the set of CHCs is non linear. When a set of CHCs is satisfiable $($ modulo $\mathcal{T}$ ), a solution is a model, including an interpretation of all the predicates in $P$, that satisfies all the CHCs. Typically, we are interested in solutions that are expressed as formulas over some class of formulas $\mathcal{A}$ [Bjørner et al. 2015]:

Definition 3.1 ( $\mathcal{A}$-Solutions). Let $\mathcal{A}$ be a class of formulas. An $\mathcal{A}$-solution for a set of CHCs $C$ assigns to each predicate symbol $p \in P$ a formula $\theta_{p}(\bar{y}) \in \mathcal{A}$ whose free variables $\bar{y}$ correspond to the arguments of $p$ such that replacing each predicate application $p\left(\bar{x}_{p}\right)$ in $C$ by $\theta_{p}\left[\bar{y} \mapsto \bar{x}_{p}\right]$ results in a valid closed formula (modulo the background theory).

It is possible that a set of CHCs is satisfiable, but has no $\mathcal{A}$-solution.

Programs as CHCs. A set of $\mathrm{CHCs}$ can encode program semantics. A non-recursive program is encoded using linear $\mathrm{CHCs}$. All linear $\mathrm{CHCs}$ are reducible to a set of $\mathrm{CHCs}$ with a single unknown predicate $I n v$ and exactly 3 clauses (free variables are implicitly universally quantified):

$$
\operatorname{Init}(\bar{x}) \Rightarrow \operatorname{Inv}(\bar{x}) \quad \operatorname{Inv}(\bar{x}) \wedge \operatorname{Tr}\left(\bar{x}, \bar{x}^{\prime}\right) \Rightarrow \operatorname{Inv}\left(\bar{x}^{\prime}\right) \quad \operatorname{Inv}(\bar{x}) \wedge \operatorname{Bad}(\bar{x}) \Rightarrow \perp
$$

In the context of safety verification, assignments to $\bar{x}$ are called states, Init represents the set of initial states of the program, $\operatorname{Tr}$ represents its set of transitions and Bad represents a set of bad states. In this case, a solution for Inv is an inductive invariant that proves that the program is safe: no bad state is reachable from an initial state via the program's transitions. When the CHC is unsatisfiable, there exists a counterexample to safety. Counterexamples to the safety property are derived from resolution proofs of unsatisfiability of CHCs. Recursive programs (and their safety) are encoded as non linear CHCs. In this case, a solution to the $\mathrm{CHCs}$ contain necessary function summaries to prove that the program is safe. Similarly, counterexamples to safety are derived from resolution proofs of unsatisfiability.

\section{CHCS MODULO ADT AND CATAMORPHISMS}

In this work, we are interested in verifying safety of programs specified using the theory of ADT and whose correctness arguments are specified using recursively defined functions. Specifically, we are interested in catamorphisms. In this section, we define catamorphisms (Sec. 4.1), and explain the conventions we use when considering CHCs that encode safety of programs (Sec. 4.2). 


\subsection{Catamorphisms}

Recursive functions are in general not expressible in First Order Logic. In SMT they can be specified by function axioms, which can precisely capture the definition of the recursion, but relax the least-fixpoint semantics (e.g., [Löding et al. 2018]). A function axiom is a formula of the form $\forall \bar{x} \cdot f(\bar{x})=t(\bar{x})$ where $t$ is a term of an appropriate sort. $t$ can contain $f$ itself as well as other function (or constant) symbols. We call a function symbol $f$ for which a function axiom is given, a recursively defined function (RDF), to distinguish it from other uninterpreted function symbols However, there is really no easy way to tell when a function axiom defines a function. Some function axioms are unsatisfiable, for example, $\forall x \cdot f(x)=\neg f(x)$. Some axioms provide a complete definition of a function, e.g., $\forall x \cdot f(x)=$ ite $(x \leq 0,0, x \times f(x-1))$, which has a unique interpretation over the standard model of arithmetic, and some do not, e.g., $\forall x \cdot f(x)=f(x+1)-1$, which is satisfied by the interpretation $f(x)=x$, but also by $f(x)=x+1, f(x)=x+2$, etc. While SMT-LIB does not restrict function axioms to uniquely define a function, our work addresses RDFs that are uniquely defined by their axioms. To avoid the need to characterize such functions, we focus on the special case of catamorphisms. In Sec. 5 we discuss how to extend our results to other well defined functions.

Catamorphisms are a notable example of function axioms that specify well defined functions over ADTs. A catamorphism (or, generalized fold) is an RDF that abstracts the content of an ADT into a single value. Common abstractions, such as length of a list, height of a tree, set of all elements of a tree, are all examples of catamorphisms. Given an interpreted sort $r$ for the range of the abstraction, a catamorphism from the list ADT List $t_{s}$ to $r$ is specified using a base case value, $b$, of sort $\mathrm{r}$, and a term, denoted $y \oplus z$, of sort $\mathrm{r}$, where $y$ and $z$ are variables of sorts $\mathrm{s}$ and $\mathrm{r}$, respectively, and all symbols are interpreted. Intuitively, the base case value $b$ is used to define the value of the catamorphism for nil, while $y \oplus z$ is used to define the value in the recursive case of a list $x$ created by the insert constructor, in which case the value is defined by applying $\oplus$ on the element $y=\operatorname{head}(x)$ inserted to the list and on the value $z$ recursively defined for tail $(x)$. Formally, given $b, \oplus$, the following function axiom defines $f$ as a catamorphism over List $_{s}$ :

$$
\forall x \cdot f(x)=\operatorname{ite}(x=\operatorname{nil}, b, \operatorname{head}(x) \oplus f(\operatorname{tail}(x)))
$$

We denote such a function axiom $\varphi_{(b, \oplus)}$ and call it a catamorphism axiom for $f$.

Example 4.1. The length of a list is a catamorphism from List to Int specified by:

$$
\varphi_{(0,+1)} \triangleq \forall x \cdot \operatorname{length}(x)=\operatorname{ite}(x=\operatorname{nil}, 0,1+\operatorname{length}(\operatorname{tail}(x))
$$

The theory of ADTs with catamorphisms (and, more generally, RDFs) is undecidable.

\subsection{CHCs Modulo ADTs and Catamorphisms}

In this section, we present the form of CHCs we are interested in. We also list out a number of assumptions so that the presentation in later parts of the paper is easier to follow. We encode programs that manipulate datatypes as CHCs modulo a theory $\mathcal{T}$ that includes the theory of ADTs. The encoding further uses catamorphisms, specified via function axioms. In the sequel, we fix a signature $\Sigma$ that consists of the sorts s, $r$, and List ${ }_{s}$, and constructors, selectors, and testers for lists. The signature $\Sigma$ also includes a function symbol $f$ of sort List s $_{\mathrm{s}} \mapsto \mathrm{r}$ that is used to specify a catamorphism. We denote by $\varphi_{(b, \oplus)}$ the catamorphism axiom for $f$. 
In the paper, we consider satisfiability of $C_{f} \wedge \varphi_{(b, \oplus)}$, where $C_{f}$ is a set of CHCs of the form:

$$
\begin{aligned}
& \operatorname{Init}(\bar{y}, \bar{z}) \wedge\left(\bigwedge_{i} f\left(y_{i}\right)=z_{i}\right) \Rightarrow \operatorname{Inv}\left(\bar{y}^{\prime}, \bar{z}^{\prime}\right) \\
& \operatorname{Inv}(\bar{y}, \bar{z}) \wedge \operatorname{Tr}\left(\bar{y}, \bar{z}, \bar{y}^{\prime}, \bar{z}^{\prime}\right) \wedge\left(\bigwedge_{i} f\left(y_{i}\right)=z_{i} \wedge f\left(y_{i}^{\prime}\right)=z_{i}^{\prime}\right) \Rightarrow \operatorname{Inv}\left(\bar{y}^{\prime}, \bar{z}^{\prime}\right) \\
& \operatorname{Inv}(\bar{y}, \bar{z}) \wedge\left(\bigwedge_{i} f\left(y_{i}\right)=z_{i}\right) \wedge \operatorname{Bad}(\bar{y}, \bar{z}) \Rightarrow \perp
\end{aligned}
$$

where $y_{i} \in \bar{y}, z_{i} \in \bar{z}, y_{i}$ is of sort List ${ }_{\mathrm{s}}$ and $z_{i}$ is of sort $\mathrm{r}$. Repetitions are allowed: $y_{i}$ can be the same as $y_{j}$. We further assume that Init, $T r$, and Bad do not contain any $f$ applications, hence the bodies of the CHCs are $f$-pure. Such a form may be obtained by purification. Inv $\notin \Sigma$ is the only unknown predicate. Throughout the paper, we use $C_{f}$ to refer to CHCs of the form Eq. (2), and we refer to $C_{f} \wedge \varphi_{(b, \oplus)}$ as CHCs modulo function axiom.

$\mathrm{CHCs}$ like those in Eq. (2) can be directly constructed from functional programs and imperative programs.

The assumptions about the sort of $y_{i}, z_{i}, f$, that there is only one ADT, and one $f$ are for presentation purposes only. We stress that our ideas and our implementation work for multiple catamorphisms defined over any ADT, mutually recursive ADTs, mutually recursive catamorphisms, and non-linear CHCs. Relaxing these assumptions does not require any new algorithmic insights, and, in the implementation required no change from the underlying $\mathrm{CHC}$ solver.

Solutions. In this paper, we limit our attention to $\mathcal{A}$-solutions of $C_{f}$ modulo $\varphi_{(b b, \oplus)}$, where $\mathcal{A}$ is the class of quantifier-free formulas over the signature $\Sigma \backslash\{f\}$. That is, $f$ is not allowed to appear in the solution. We leave lifting this assumption to future work. Solutions that involve $f$ can be accounted for, even with the restricted language, if the CHCs are constructed in such a way that the constraints in the clauses contain all the necessary $f$ applications to prove safety (i.e., to obtain a solution), and that these applications are captured by variables that are included as arguments to solutions. In particular, term abstraction [Alberti et al. 2012] may be used to introduce auxiliary variables that represent $f$-applications that are needed in order to express the solution, and augment the bodies of the CHCs with them. For example, in Fig. 2, the variable $j$ that is added as an argument to Inv is an auxiliary variable introduced to express length(y).

Motivation for our work. Existing solvers for $\mathrm{CHCs}$ make multiple satisfiability queries over the background theory. Unfortunately, satisfiability becomes undecidable when considering the theory of ADTs with RDFs. The remainder of the paper is dedicated to showing how existing solvers may be extended to handle such $\mathrm{CHCs}$ while ensuring that all satisfiability queries remain decidable.

\section{REDUCING CHCS MODULO CATAMORPHISMS TO CHCS}

To alleviate the problems arising from undecidability of catamorphisms, we show how to reduce $\mathrm{CHCs}$ modulo catamorphisms to pure $\mathrm{CHCs}$ whose underlying theory is decidable. Specifically, we show how to reduce $C_{f} \wedge \varphi_{(b, \oplus)}$, a set of CHCs with an axiomatized catamorphism $f$, to an equisatisfiable set of CHCs $C_{F}$ without $f$. The key idea is to encode $f$ by a fresh predicate (relation) symbol $F$, of appropriate sort, i.e., if $f$ is of sort List $_{\mathrm{s}} \mapsto \mathrm{r}$, then $F$ is a predicate over sorts List $\mathrm{s}_{\mathrm{s}} \times \mathrm{r}$. The predicate $F$ is added to the set of unknown CHC predicates (which in our case consists of Inv only), and is used instead of $f$ in $C_{f}$. The challenge is to express the axiom for $f\left(\varphi_{(b, \oplus)}\right)$ as a CHC over $F$, while preserving equisatisfiability.

From $\mathrm{CHCs}$ with $f$ to $\mathrm{CHCs}$ with $\mathrm{F}$. We first address the $\mathrm{CHCs} C_{f}$. As the bodies of these $\mathrm{CHCs}$ are $f$-pure, we can use a simple substitution to replace $f$ by $F$. 
Definition 5.1 (Relationification). Let $C_{f}$ be a set of CHCs whose bodies are $f$-pure. We denote by $C_{f}[f \mapsto F]$ the set of CHCs obtained by replacing all equalities of the form $f(t)=z$ in the constraints of $C_{f}$, with $F(t, z)$.

The $\mathrm{CHCs}$ resulting from relationification do not contain any occurrences of the function symbol $f$. On the other hand, they contain the new unknown predicate $F$. For example, relationification of the $\mathrm{CHC} \forall x, y, z \cdot A(x) \wedge f(x)=y \wedge f(y)=z \Rightarrow B(z)$ gives us $\forall x, y, z \cdot A(x) \wedge F(x, y) \wedge F(y, z) \Rightarrow B(z)$

The following lemma outlines the conditions in which relationification preserves satisfiability:

LEMMA 5.2. Let $M$ be a model, and $f$ and $F$ be interpretations of $f$ and $F$ over the universe of $M$, respectively.

- If $M[f \mapsto f] \vDash C_{f}$ and $\boldsymbol{F}$ is the graph of $f$ then $M[F \mapsto F] \vDash C_{f}[f \mapsto F]$.

- If $M[F \mapsto F] \vDash C_{f}[f \mapsto F]$ and $\boldsymbol{F}$ is an over approximation of the graph of $f$ then $M[f \mapsto$ $f] \vDash C_{f}$.

Proof. The first statement is straight forward. For the second statement, we have $M[F \mapsto$ $F] \vDash C_{f}[f \mapsto F]$. Let $G_{f}$ be a stronger relation than $\boldsymbol{F}$. Since $F$ occurs only on the tail of clauses in $C_{f}[f \mapsto F]$, we have that $M\left[F \mapsto G_{f}\right] \vDash C_{f}[f \mapsto F]$. In particular, $G_{f}$ can be the relation $\{(x, z) \mid f(x)=z\}$, i.e., the graph of $f$. This implies that $M[f \mapsto f] \vDash C_{f}$.

From function axioms to CHCs. Now, we move on to constructing CHCs to capture the catamorphism axiom.

Definition 5.3 (Positive relation constraint). Given a catamorphism axiom $\varphi_{(b, \oplus)}$ for $f$ and a fresh relation symbol $F$, the positive relation constraint for $\varphi_{(b, \oplus)}$ is

$$
R C_{F}^{+}\left(\varphi_{(b, \oplus)}\right)=\left(\begin{array}{c}
\forall x, z \cdot x=\operatorname{nil} \wedge z=b \Rightarrow F(x, z) \wedge \\
\forall x, l, z \cdot x \neq \operatorname{nil} \wedge F(\operatorname{tail}(x), l) \wedge z=l \oplus \operatorname{head}(x) \Rightarrow F(x, z)
\end{array}\right)
$$

The positive relation constraint corresponding to a catamorphism definition consists of one $\mathrm{CHC}$ for the base case and one $\mathrm{CHC}$ for the recursive case.

Example 5.4. Consider the axiom for the catamorphism length, $\varphi_{(0,+1)}$, from Ex. 4.1. Let Length be a fresh relation symbol. The positive relation constraint for $\varphi_{(0,+1)}$ is:

$$
\begin{aligned}
\forall x, z \cdot x=\operatorname{nil} \wedge z=0 & \Rightarrow \operatorname{Length}(x, z) \wedge \\
\forall x, l, z \cdot x \neq \operatorname{nil} \wedge \operatorname{Length}(\operatorname{tail}(x), l) \wedge z=l+1 & \Rightarrow \operatorname{Length}(x, z)
\end{aligned}
$$

Despite seemingly capturing only one direction of the definition encapsulated in the catamorphism axiom $\varphi_{(b, \oplus)}$, Thm. 5.7 shows that the positive relation constraint $R C_{F}^{+}\left(\varphi_{(b, \oplus)}\right)$ does not allow spurious solutions when $F$ is used instead of $f$ in $C_{f}$, hence preserving satisfiability in both directions. This results from the following lemma that ensures that $f$ is always a solution to $R C_{F}^{+}\left(\varphi_{(b, \oplus)}\right)$ and that all solutions must overapproximate $f$.

LEMMA 5.5 ( $F$ OVER APPROXIMATES $f$ ). Let $M$ be a model and $f$ an interpretation of $f$ over the universe of $M$. If $M[f \mapsto f] \vDash \varphi_{(b, \oplus)}$ then

a) The relation $F$ over the universe of $M$ defined by the graph of $f$ satisfies $M[F \mapsto F] \vDash$ $R C_{F}^{+}\left(\varphi_{(b, \oplus)}\right)$.

b) For every relation $\boldsymbol{F}$ over the universe of $M$, if $M[F \mapsto F] \vDash R C_{F}^{+}\left(\varphi_{(b, \oplus)}\right)$ then $\boldsymbol{F}$ is an over approximation of the graph of $f$.

\footnotetext{
${ }^{2}$ The graph of a function $f$ is the relation $F=\{(x, f(x)) \mid x \in \operatorname{dom}(f)\}$, where $\operatorname{dom}(f)$ is the domain of $f$.
} 


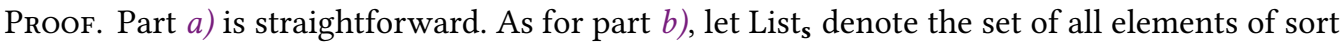
List $_{\mathrm{s}}$ in the universe of $M$. Wlog, we can assume that List ${ }_{s}$ consists of all constructor terms (see Sec. 3.2). Therefore, we can prove $b$ ) by straight forward induction on the elements of List s $_{\mathbf{s}}$ For the base case, since $f(\boldsymbol{n i l})=\boldsymbol{b}$, we have to show that $(\boldsymbol{n} \boldsymbol{i l}, \boldsymbol{b}) \in \boldsymbol{F}$. We can see that the clause $\forall x, z \cdot x=$ nil $\wedge b=z \Rightarrow F(x, z)$ enforces this. For the recursive case, assume that $f(y)=\boldsymbol{l}$ and $(\boldsymbol{y}, \boldsymbol{l}) \in \boldsymbol{F}$. Let $\boldsymbol{x}=\boldsymbol{i n s e r t}(\boldsymbol{k}, \boldsymbol{y})$ and $f(\boldsymbol{x})=\boldsymbol{z}$. It has to be the case that $z=\boldsymbol{k} \oplus \boldsymbol{l}$. The clause $\forall x, l, z \cdot x \neq \operatorname{nil} \wedge F(\operatorname{tail}(x), l) \wedge z=$ head $(x) \oplus l \Rightarrow F(x, z)$ ensures that $(x, z) \in F$. Thus, all tuples $(x, z)$ such that $f(x)=z$ must belong to the relation $F$.

We are now ready to define the CHCs obtained after eliminating $f$ :

Definition 5.6 (Relationified CHCs with CHC encoding of catamorphisms). Given $C_{f}$ and $\varphi_{(b, \oplus)}$, the set of $\mathrm{CHCs} C_{F}$ obtained by relationification of $C_{f}$ and reducing the function axiom $\varphi_{(b, \oplus)}$ to $\mathrm{CHCs}$ is

$$
C_{F}=R C_{F}^{+}\left(\varphi_{(b, \oplus)}\right) \wedge C_{f}[f \mapsto F]
$$

THEOREM 5.7. The set of CHCs after the transformation is equisatisfiable to the original CHCs modulo catamorphisms: $C_{F} \approx \varphi_{(b, \oplus)} \wedge C_{f}$.

Proof. Left to right direction. Let $M$ be a model for $R C_{F}^{+}\left(\varphi_{(b, \oplus)}\right) \wedge C_{f}[f \mapsto F]$, and denote $M[F]$ by $\boldsymbol{F}$. Let $f$ be a function over the universe of $M$ such that $M[f \mapsto f] \vDash \varphi_{(b, \oplus)} . f$ exists because $\varphi_{(b b, \oplus)}$ defines a catamorphism. We know from Lem. 5.5 that $\boldsymbol{F}$ over approximates the graph of $\boldsymbol{f}$. Therefore, by Lem. 5.2 we can see that $M[f \mapsto f]$ is a model for $\varphi_{(b, \oplus D} \wedge C_{f}$ as well.

Right to Left direction. Let $M$ be a model for $\varphi_{(b, \oplus)} \wedge C_{f}$. Let $M[f]=f$ and let $F$ be the graph of $\boldsymbol{f}$. By Lem. 5.5, we know that $M[F \mapsto \boldsymbol{F}] \vDash R C_{F}^{+}\left(\varphi_{(b, \oplus)}\right)$. Therefore, by Lem. 5.2, $M[F \mapsto \boldsymbol{F}] \vDash$ $C_{f}[f \mapsto F]$.

Applying the transformation on $\mathrm{CHCs} C_{f}$ of the form given in Eq. (2), we get:

$$
\begin{aligned}
y=\operatorname{nil} \wedge z=b & \Rightarrow F(y, z) \\
y \neq \operatorname{nil} \wedge F(\operatorname{tail}(y), l) \wedge y=l \oplus \operatorname{head}(x) & \Rightarrow F(y, z) \\
\operatorname{Init}(\bar{y}, \bar{z}) \wedge\left(\bigwedge_{i} F\left(y_{i}, z_{i}\right)\right) & \Rightarrow \operatorname{Inv}\left(\bar{y}^{\prime}, \bar{z}^{\prime}\right) \\
\operatorname{Inv}(\bar{y}, \bar{z}) \wedge \operatorname{Tr}\left(\bar{y}, \bar{z}_{,}, \bar{y}^{\prime}, \bar{z}^{\prime}\right) \wedge\left(\bigwedge_{i} F\left(y_{i}, z_{i}\right) \wedge F\left(y_{i}^{\prime}, z_{i}^{\prime}\right)\right) & \Rightarrow \operatorname{Inv}\left(\bar{y}^{\prime}, \bar{z}^{\prime}\right) \\
\operatorname{Inv}(\bar{y}, \bar{z}) \wedge\left(\bigwedge_{i} F\left(y_{i}, z_{i}\right)\right) \wedge \operatorname{Bad}(\bar{y}, \bar{z}) & \Rightarrow \perp
\end{aligned}
$$

While the transformation preserves satisfiability, the following example demonstrates that it may not preserve $\mathcal{A}$-solutions (Def. 3.1):

Example 5.8 (The transformation does not preserve solutions). The set of CHCs in Eq. (5) encode the safety of a program that removes all the elements from a list $y$ while keeping track of its length $i$. We use term abstraction to encode length $(y)$ via an auxiliary integer variable $j$, such that neither $y$ nor length $(y)$ are needed in the inductive invariant. Accordingly, the inductive invariant is specified over $i, j$ (note that $y$ is also omitted from Inv in this example). The first clause initializes variable $i$ to the length of $y$ and the second clause keeps track of the length of $y$ as elements are removed from the list. The third clause derives false if $i$ is non zero once all elements are removed.

$$
\begin{aligned}
\operatorname{length}(y)=i & \Rightarrow \operatorname{Inv}(i, i) \\
\operatorname{Inv}(i, j) \wedge \operatorname{length}(y)=j \wedge y \neq \operatorname{nil} \wedge y^{\prime}=\operatorname{tail}(y) \wedge \operatorname{length}\left(y^{\prime}\right)=j^{\prime} & \Rightarrow \operatorname{Inv}\left(i-1, j^{\prime}\right) \\
\operatorname{Inv}(i, j) \wedge \operatorname{length}(y)=j \wedge y=\operatorname{nil} \wedge i \neq 0 & \Rightarrow \perp
\end{aligned}
$$


The set of CHCs in Eq. (5) is satisfiable modulo $\varphi_{(0,+1)}$, the catamorphism axiom for length, with the solution: $\operatorname{Inv}(i, j)=i=j$, defined over a signature that excludes length. The equi-satisfiable CHC $C_{F}=R C_{F}^{+}\left(\varphi_{(0,+1)}\right) \wedge C[$ length $\mapsto$ Length $]$ is

$$
\begin{aligned}
y=\operatorname{nil} \wedge z=0 & \Rightarrow \operatorname{Length}(y, z) \\
y \neq \operatorname{nil} \wedge \operatorname{Length}(\operatorname{tail}(y), l) \wedge z=1+l & \Rightarrow \operatorname{Length}(y, z) \\
\operatorname{Length}(y, i) & \Rightarrow \operatorname{Inv}(i, i) \\
\operatorname{Inv}(i, j) \wedge \operatorname{Length}(y, j) \wedge y \neq \operatorname{nil} \wedge y^{\prime}=\operatorname{tail}(y) \wedge \operatorname{Length}\left(y^{\prime}, j^{\prime}\right) & \Rightarrow \operatorname{Inv}\left(i-1, j^{\prime}\right) \\
\operatorname{Inv}(i, j) \wedge \operatorname{Length}(y, j) \wedge y=\operatorname{nil} \wedge i \neq 0 & \Rightarrow \perp
\end{aligned}
$$

However, it does not have a solution in which the interpretation for Length is expressible without length. Intuitively, this is because the interpretation for Length in a solution needs to be exactly the graph of the interpretation of length, while the relation corresponding to length cannot be expressed in first order logic. We formalize this intuition next. Assume to the contrary that there is a model $M$ for the transformed CHCs in which the interpretation of Length, Length, is not equal to the graph of length, where length is the (unique) interpretation of length over the universe of $M$. Then, Length has to be an over approximation of the graph of length (due to Lem. 5.5). Take a pair $(\boldsymbol{y}, \boldsymbol{j})$ that is in Length but not in the graph of length, i.e., length $(\boldsymbol{y}) \neq \boldsymbol{j}$, such that $\boldsymbol{y}$ is minimal. We show that the last $\mathrm{CHC}$ must be violated. If $\boldsymbol{y}=\boldsymbol{n i l}$, use the third clause to deduce that $(\boldsymbol{j}, \boldsymbol{j}) \in \boldsymbol{I} \boldsymbol{n} \boldsymbol{v}$, which immediately implies that the last clause is violated (since by our assumption $\boldsymbol{j} \neq \mathbf{0}$ in this case). Otherwise, consider $\boldsymbol{y}^{\prime}=\boldsymbol{t a i l}(\boldsymbol{y})$ and $\boldsymbol{j}^{\prime}=\boldsymbol{l e n g t h}\left(\boldsymbol{y}^{\prime}\right)$. Since Length overapproximates length, $\left(\boldsymbol{y}^{\prime}, \boldsymbol{j}^{\prime}\right) \in$ Length as well. From the $4^{\text {th }}$ clause, we deduce that $\left(\boldsymbol{j}-\mathbf{1}, \boldsymbol{j}^{\prime}\right) \in \mathbf{I n} \boldsymbol{v}$, even though $\boldsymbol{j}-\mathbf{1} \neq \boldsymbol{j}^{\prime}$ (recall that $\boldsymbol{j} \neq \boldsymbol{\operatorname { l e n g t h }}(\boldsymbol{y})$ whereas length $\left.(y)=\operatorname{length}\left(\boldsymbol{y}^{\prime}\right)+\mathbf{1}=\boldsymbol{j}^{\prime}+1\right)$. From $\left(\boldsymbol{j}-\mathbf{1}, \boldsymbol{j}^{\prime}\right) \in \mathbf{I n} \boldsymbol{v}$, using the pairs in the graph of length, we can use the $4^{\text {th }}$ clause to iteratively decrease both elements of the pair by 1, ultimately showing that there exists $i \neq 0$ such that $(\boldsymbol{i}, \mathbf{0}) \in \mathbf{I n} \boldsymbol{v}$. This again contradicts the last clause (by taking the pair (nil, 0$) \in$ Length).

REMARK. We have shown how to relationify catamorphisms on lists. Extending this to catamorphisms on other ADTs is straightforward: relationification of $f$ introduces a Horn clause over the uninterpreted predicate $F$ for each of the base and recursive cases.

The approach also works for RDFs over ADTs that are defined using SMT-LIB syntax. In this case, an RDF is defined by a single axiom: $\forall \bar{v} \cdot f(\bar{v})=t(\bar{v})$. To perform relationification, we further require that the base and recursive cases can be easily identified. This is already used by the majority of SMT-solvers supporting RDFs. For soundness, we require that the axiom is satisfiable (i.e., actually defines a function). This requirement is omitted from SMT-LIB, but is common in many other systems that allow user-defined recursive functions (e.g., Dafny [Leino 2010]). In general, our approach applies to any RDF that supports an appropriate relationification procedure satisfying Lem. 5.5.

To conclude, in this section, we presented a transformation that takes as input a set of CHCs modulo RDFs, $C_{f} \wedge \varphi_{(b, \oplus)}$, and produces a set of CHCs without RDFs, $C_{F}$. The transformation preserves satisfiability (Thm. 5.7) but not solutions (Ex. 5.8). Note that all solutions to $C_{F}$ are solutions to $C_{f} \wedge R C_{F}^{+}\left(\varphi_{(b, \oplus)}\right)$.

\section{INTRODUCING RDF ABSTRACTIONS INTO CHCS}

In this section we tackle the potential loss of $\mathrm{CHC}$ solutions without compromising decidability of the background theory by using abstractions of the RDFs.

We start by re-introducing the RDF with the axiom that defines it, thus recovering solutions. Formally, instead of substituting $f(x)=z$ by $F(x, z)$ during relationification, we substitute it by $f(x)=z \wedge F(x, z)$. Let $C_{f}[f \mapsto(f \wedge F)]$ denote this transformation. 
Definition 6.1 (Relationified CHCs modulo RDFs). Given $C_{f}$ and $\varphi_{(b, \oplus)}$, the set of CHCs $C_{f F}$ obtained after relationification, reducing function axiom $\varphi_{(b, \oplus)}$ to $\mathrm{CHCs}$ and re-introducing RDFs is

$$
C_{f F}=R C_{F}^{+}\left(\varphi_{(b, \oplus)}\right) \wedge C_{f}[f \mapsto(f \wedge F)]
$$

Specifically, when $C_{f}$ is Eq. (4) modulo $\varphi_{(b, \oplus)}, C_{f F}$ is,

$$
\begin{aligned}
\psi_{b}(y, z) & \Rightarrow F(y, z) \\
\psi_{r}(y, z)[f \mapsto F] & \Rightarrow F(y, z) \\
\operatorname{Init}(\bar{y}, \bar{z}) \wedge\left(\bigwedge_{i} F\left(y_{i}, z_{i}\right) \wedge f\left(y_{i}\right)=z_{i}\right) & \Rightarrow \operatorname{Inv}\left(\bar{y}^{\prime}, \bar{z}^{\prime}\right) \\
\operatorname{Inv}(\bar{y}, \bar{z}) \wedge \operatorname{Tr}\left(\bar{y}, \bar{z}, \bar{y}^{\prime}, \bar{z}^{\prime}\right) \wedge\left(\bigwedge_{i} F\left(y_{i}, z_{i}\right) \wedge f\left(y_{i}\right)=z_{i} \wedge F\left(y_{i}^{\prime}, z_{i}^{\prime}\right) \wedge f\left(y_{i}^{\prime}\right)=z_{i}^{\prime}\right) & \Rightarrow \operatorname{Inv}\left(\bar{y}^{\prime}, \bar{z}^{\prime}\right) \\
\operatorname{Inv}(\bar{y}, \bar{z}) \wedge\left(\bigwedge_{i} F\left(y_{i}, z_{i}\right) \wedge f\left(y_{i}\right)=z_{i}\right) \wedge \operatorname{Bad}(\bar{y}, \bar{z}) & \Rightarrow \perp
\end{aligned}
$$

This transformation preserves not only satisfiability, but also solutions, in the following sense:

Theorem 6.2. If $M \vDash \varphi_{(b, \oplus)} \wedge C_{f}$ then $M[F \mapsto \mathrm{T}] \vDash \varphi_{(b, \oplus)} \wedge C_{f F}$. If $M \vDash \varphi_{(b, \oplus)} \wedge C_{f F}$, then $M \vDash \varphi_{(b, \oplus)} \wedge C_{f}$

Proof. First part is straightforward; second part follows from $M[f] \subseteq M[F]$ (Lem. 5.5).

Corollary 6.3. $\varphi_{(b, \oplus)} \wedge C_{f} \approx \varphi_{(b, \oplus)} \wedge C_{f F}$.

From Thm. 6.2 we can see that, if there is an $\mathcal{A}$-solution to $\varphi_{(b, \oplus)} \wedge C_{f}$ then there will also be an $\mathcal{A}$-solution to $\varphi_{(b, \oplus)} \wedge C_{f F}$ (assuming that $\left.\mathrm{T} \in \mathcal{A}\right)$.

Unfortunately, re-introducing the catamorphisms with their axioms also makes the underlying logic undecidable. To mitigate this, we work over abstractions of the catamorphism. In the remainder of the section, we define the abstractions we use (Sec. 6.1) and explain what impact the abstractions have on the solutions to the CHCs (Sec. 6.2). In Sec. 7, we describe an algorithm for checking the satisfiability of CHCs modulo RDFs using these abstractions.

\subsection{Abstraction of RDF}

In this section, we define two abstractions of formulas modulo RDFs. The first abstraction unfolds $\mathrm{RDF}$ applications using the axioms of RDF and then replaces any remaining RDF applications with uninterpreted functions and the second abstraction further replaces the literals containing uninterpreted functions with $T$. Both of the abstractions result in weaker formulas, and can be understood as over approximating the formulas to which they are applied. As we will see later, these abstractions allow us to stay in a decidable fragment when validating solutions, while guaranteeing that we have potentially more solutions than $C_{F}$.

To define the abstractions precisely, we first define prenex $f$-pure formulas. A formula is prenex $f$-pure if it is of the form

$$
\exists \bar{z} \cdot\left(\bigwedge_{i} f\left(e_{i}\right)=z_{i}\right) \wedge \theta^{\prime}(\bar{x}, \bar{z})
$$

where each $e_{i}$ is a term over free variables $\bar{x}$, variables $\bar{z}$, and containing no $f$, each $z_{i}$ is a variable in $\bar{z}$, and $\theta^{\prime}$ is a quantifier free formula over $\bar{x}$ and $\bar{z}$ containing no $f$ terms. Every quantifier-free formula $\theta$ can be converted into an equivalent prenex $f$-pure formula by the purification procedure described in Sec. 3: introducing existentially quantified variables that represent the $f$ applications. For example, $p(f(f(x)))$ may be rewritten into $\exists y, z \cdot f(x)=y \wedge f(y)=z \wedge p(z)$. 
Instantiating function axiom. Given an $\operatorname{RDF} f$ together with its function axiom $\varphi=\forall x \cdot f(x)=$ $t(x)$, and a prenex $f$-pure formula $\theta$, instantiation of $f$ in $\theta$ is the formula obtained by conjoining the literal $t\left[x \mapsto e_{i}\right]=z_{i}$ with each literal $f\left(e_{i}\right)=z_{i}$ in $\theta$. The $k$-instantiation of $f$ on $\theta$ is the formula defined recursively as follows: the 0 -instantiation is $\theta$ itself and $(i+1)$-instantiation is the instantiation of $f$ on the $i$-instantiation (after the $i$-instantiation is brought to prenex $f$-pure form)

Definition 6.4 ( $k$-instantiation abstraction). Given an $\operatorname{RDF} f$ and a prenex $f$-pure formula $\theta$, the $k$-instantiation abstraction of $f$ on $\theta$, denoted by $\alpha_{r f}^{k}(\theta)$, is the formula obtained by replacing all $f$ occurrences in the $k$-instantiation of $\theta$ with a fresh uninterpreted function.

Example 6.5. Let $\theta=$ length $(x)=z_{1} \wedge$ length $(y)=z_{2} \wedge y=\operatorname{tail}(x) \wedge z_{1}=2 \wedge z_{2} \neq 1$. Then,

$$
\begin{aligned}
& \alpha_{r f}^{0}(\theta)=\text { length }_{u f}(x)=z_{1} \wedge \text { length } h_{u f}(y)=z_{2} \wedge y=\operatorname{tail}(x) \wedge z_{1}=2 \wedge z_{2} \neq 1 \\
& \alpha_{r f}^{1}(\theta)=\alpha_{r f}^{0}(\theta) \wedge \text { ite }\left(x=\text { nil, } 0,1+\text { length }_{u f}(\operatorname{tail}(x))\right)=z_{1} \wedge \\
& \text { ite }\left(y=n i l, 0,1+\text { length }_{u f}(\operatorname{tail}(y))\right)=z_{2} \\
& \alpha_{r f}^{2}(\theta)=\exists l_{t x}, l_{t y} \cdot \text { length }_{u f}(\operatorname{tail}(x))=l_{t x} \wedge \text { length }_{u f}(\operatorname{tail}(y))=l_{t y} \wedge \\
& \alpha_{r f}^{0}(\theta) \wedge i t e\left(x=n i l, 0,1+l_{t x}\right)=z_{1} \wedge \text { ite }\left(y=n i l, 0,1+l_{t y}\right)=z_{2} \wedge \\
& \text { ite }\left(\operatorname{tail}(x)=n i l, 0,1+\text { length }_{u f}(\operatorname{tail}(\operatorname{tail}(x)))\right)=l_{t x} \wedge \\
& \text { ite }\left(\operatorname{tail}(y)=n i l, 0,1+\text { length }_{u f}(\operatorname{tail}(\operatorname{tail}(y)))\right)=l_{t y}
\end{aligned}
$$

Definition 6.6 (Uninterpreted function abstraction). Given an uninterpreted function symbol $f$, and a prenex $f$-pure formula $\theta$, the uninterpreted function abstraction, denoted $\alpha^{\uparrow}(\theta)$, is obtained by replacing all literals containing $f$ in $\theta$ with $\mathrm{T}$.

We write $\alpha_{r f}^{k \uparrow}(\theta)$ for $\left(\alpha_{r f}^{\uparrow} \circ \alpha_{r f}^{k}\right)(\theta)$.

Example 6.7. The result of applying the uninterpreted function abstraction for the $k$-unrolling abstractions in Ex. 6.5 is

$$
\begin{aligned}
\alpha_{r f}^{0 \uparrow}(\theta)= & y=\operatorname{tail}(x) \wedge z_{1}=2 \wedge z_{2} \neq 1 \\
\alpha_{r f}^{1 \uparrow}(\theta)= & \exists l_{t x}, l_{t y} \cdot \alpha_{r f}^{0 \uparrow}(\theta) \wedge i t e\left(x=n i l, 0,1+l_{t x}\right)=z_{1} \wedge i t e\left(y=n i l, 0,1+l_{t y}\right)=z_{2} \\
\alpha_{r f}^{2 \uparrow}(\theta)= & \exists l_{t t x}, l_{t t y}, l_{t x}, l_{t y} \cdot \alpha_{r f}^{0 \uparrow}(\theta) \wedge i t e\left(x=n i l, 0,1+l_{t x}\right)=z_{1} \wedge i t e\left(y=n i l, 0,1+l_{t y}\right)=z_{2} \wedge \\
& \text { ite }\left(\operatorname{tail}(x)=n i l, 0,1+l_{t t x}\right)=l_{t x} \wedge \text { ite }\left(\operatorname{tail}(y)=n i l, 0,1+l_{t t y}\right)=l_{t y}
\end{aligned}
$$

Notice that $\alpha_{r f}^{1 \uparrow}(\theta)$ has additional, existentially quantified, variables not present in $\alpha_{r f}^{1}(\theta)$. These variables are introduced during purification. Similarly, the additional variables in $\alpha_{r f}^{2 \uparrow}(\theta)$ have also been produced during purification.

The abstractions provide overapproximations of $\theta$ in the sense that they may only increase the set of satisfying models:

LemmA 6.8. Let $f$ be an RDF, $\theta$ a prenex $f$-pure formula and $M$ a model. Then, for any $k$,

a) $M \vDash \theta \Longrightarrow M\left[f_{u f} \mapsto M[f]\right] \vDash \alpha_{r f}^{k}(\theta)$, and

b) $M \vDash \alpha_{r f}^{k}(\theta) \Longrightarrow M \vDash \alpha_{r f}^{k \uparrow}(\theta)$.

\subsection{CHCs Modulo RDF vs CHCs over RDF Abstractions}

Recall that, originally we started with a set of CHCs $C_{f}$ that contain applications of $\operatorname{RDF} f$. We showed that replacing $f$ with a fresh relation $F$ preserves satisfiability (Thm. 5.7) but not solutions (Ex. 5.8). Re-introducing $f$ regains solutions (Thm. 6.2), but also brings back the problem of 
undecidability. In this section, we analyze the effects of re-introducing abstractions of the RDF $f$ into $C_{F}$. Recall that $C_{F}=R C_{F}^{+}\left(\varphi_{(b, \oplus)}\right) \wedge C_{f}[f \mapsto F]$ is the transformed CHCs without $f$ applications, and $C_{f F}=R C_{F}^{+}\left(\varphi_{(b, \oplus)}\right) \wedge C_{f}[f \mapsto(f \wedge F)]$ is the CHCs we get after conjoining back $f$.

Using the $k$-instantiation abstraction. When trying to establish that $C_{f F}$ is satisfiable modulo $\varphi_{(b, \oplus)}$, our algorithm uses the $k$-instantiation abstraction. Let $C_{f F}^{k}$ be the result of replacing all constraints in the clauses of $C_{f F}$ with their $k$-instantiation abstractions. (The resulting formulas may be normalized so that all the newly introduced existential quantifiers in the constraints are converted to universal quantifiers over the whole clause.)

$C_{f F}^{k}$ has uninterpreted functions. This means that satisfiability of $C_{f F}^{k}$ allows the uninterpreted functions to receive some interpretation, while we are interested in solutions that are valid for any interpretation of the functions, including the ones that satisfy the abstracted function axioms (akin to an implicit second order universal quantifier over $f$ ). Therefore, $C_{f F}^{k}$ is outside of the usual domain of $\mathrm{CHCs}$, and we refrain from considering its satifiability. Instead, we extend the notion of an $\mathcal{A}$-solution (Def. 3.1) to the abstracted formula $C_{f F}^{k}$, and compare $\mathcal{A}$-solutions of $C_{f F}^{k}$ with $\mathcal{A}$-solutions of the original set of CHCs. Recall that once the solution is plugged in, the requirement is that the resulting sentences are valid, which has the effect of implicitly universally quantifying over the uninterpreted function $f$. The next theorems show that the $k$-instatiation abstractions induce a hierarchy in terms of their sets of solution.

Theorem 6.9. Let $S$ be an $\mathcal{A}$-solution to $C_{f F}^{k}$. Then, $S$ is an $\mathcal{A}$-solution to $C_{f F}$ modulo $\varphi_{(b, \oplus)}$.

Proof. Let $k C$ be the set of CHCs obtained by doing $k$-instantiation (no abstraction) of $C_{f F}$. By the properties of $k$-instantiation, we know that $k C \equiv C_{f F}$ modulo $\varphi_{(b, \oplus)}$. Let $\psi$ be the conjunction of the sentences we get from $k C$ by replacing all uninterpreted predicates with their corresponding formulas from $S$. It suffices to show that $\psi$ is valid modulo $\varphi_{(b, \oplus)}$. Similarly, let $\psi^{k}$ be the conjunction of sentences we get from $C_{f F}^{k}$ by replacing all uninterpreted predicates with their corresponding formulas from $S$. $\psi$ and $\psi^{k}$ differ only in $f$ being uninterpreted in $\psi^{k}$. Since $\psi^{k}$ is valid, $\psi$ has to be valid modulo $\varphi_{(b, \oplus)}$. Thus, $S$ is an $\mathcal{A}$-solution to $C_{f F}$ modulo $\varphi_{(b, \oplus)}$.

Importantly, validating $\mathcal{A}$-solutions to $C_{f F}^{k}$ amounts to checking validity of universally quantified formulas (equivalently, checking unsatisfiability of quantifier-free formulas) modulo the background theory combined with uninterpreted functions, which is decidable.

Another interesting aspect of $C_{f F}^{k}$ is that $\mathcal{A}$-solutions are preserved as $k$ increases:

THEOREM 6.10. If $S$ is an $\mathcal{A}$-solution to $C_{f F}^{k}$, then $S$ is an $\mathcal{A}$-solution to $C_{f F}^{k^{\prime}}$, where $k^{\prime} \geq k$.

Proof. The constraints in the clauses of $C_{f F}^{k^{\prime}}$ are stronger than the constraints in the corresponding clauses of $C_{f F}^{k}$.

Similarly, if $S$ is an $\mathcal{A}$-solution to $C_{F}$, then $S$ is an $\mathcal{A}$-solution to $C_{f F}^{k}$ for any $k$. The hierarchy induced by the different abstractions is outlined in Fig. 4. The hierarchy implies that when searching for a solution to $C_{f F}$ modulo $\varphi_{(b, \oplus)}$, we can start with $C_{f F}^{0}$ and unroll $f$ more and more, potentially increasing the set of solutions. If we find a solution for any value of $k$, we have a solution to our original problem. 


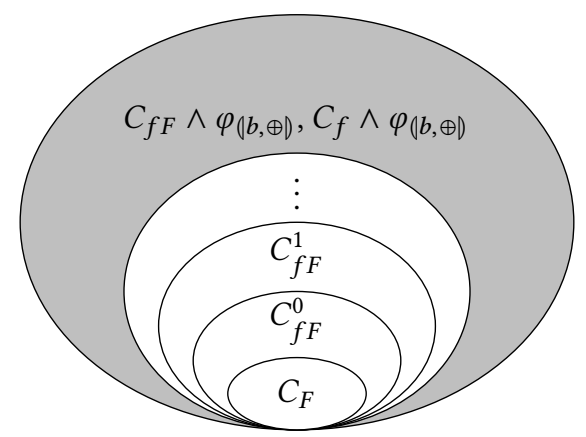

Fig. 4. Comparing $\mathcal{A}$-solutions of different abstractions of $\mathrm{CHC}$. Each ellipse represents the set of $\mathcal{A}$-solutions to the corresponding formula. Validating solutions of $\mathrm{CHC}$ in the shaded region is undecidable.

Example 6.11. Recall the set of CHCs from Ex. 5.8. We showed that the relationified $\mathrm{CHC} C_{F}$ did not have an $\mathcal{A}$-solution. However, the set of abstracted CHCs $C_{f F}^{1}$ :

$$
\begin{aligned}
& y=n i l \wedge z=0 \Rightarrow \operatorname{Length}(y, z) \\
& y \neq \operatorname{nil} \wedge \operatorname{Length}(\operatorname{tail}(y), l) \wedge z=1+l \Rightarrow \operatorname{Length}(y, z) \\
& \text { Length }(y, i) \wedge i=\operatorname{length}_{u f}(y) \wedge i=\operatorname{ite}\left(y=\operatorname{nil}, 0,1+\text { length }_{u f}(\operatorname{tail}(y))\right) \Rightarrow \operatorname{Inv}(y, i, i) \\
& \text { Length }(y, j) \wedge j=\operatorname{length}_{u f}(y) \wedge j=i \operatorname{te}\left(y=\operatorname{nil}, 0,1+\text { length }_{u f}(\operatorname{tail}(y))\right) \wedge \\
& \operatorname{Length}\left(y^{\prime}, j^{\prime}\right) \wedge j^{\prime}=\operatorname{length}_{u f}\left(y^{\prime}\right) \wedge j^{\prime}=i \operatorname{ite}\left(y^{\prime}=n i l, 0,1+\text { length }_{u f}\left(\operatorname{tail}\left(y^{\prime}\right)\right)\right) \wedge \\
& \operatorname{Inv}(y, i, j) \wedge y \neq \operatorname{nil} \wedge y^{\prime}=\operatorname{tail}(y) \Rightarrow \operatorname{Inv}\left(y^{\prime}, i-1, j^{\prime}\right) \\
& \operatorname{Length}(y, j) \wedge j=\operatorname{length}_{u f}(y) \wedge j=i \operatorname{te}\left(y=\text { nil, } 0,1+\operatorname{length}_{u f}(\operatorname{tail}(y))\right) \wedge \\
& \operatorname{Inv}(y, i, j) \wedge y=n i l \wedge i \neq 0 \Rightarrow \perp
\end{aligned}
$$

has a solution which maps Inv to the formula $i=j$ (as in the solution for $C_{f}$ ) and Length to the formula $T$. Furthermore, validating this solution is decidable as the validation query is a quantifier free formula over a combination of ADTs, LIA, and UF.

Using the uninterpreted function abstraction. When trying to establish that $C_{f F}$ is unsatisfiable modulo $\varphi_{(b, \oplus)}$, our algorithm avoids the presence of uninterpreted functions in the CHCs by using the uninterpreted function abstraction.

Let $C_{f F}^{k \uparrow}$ be the result of replacing all constraints in the clauses of $C_{f F}$ with their $\alpha_{r f}^{k \uparrow}$ abstractions. This abstraction preserves counterexamples:

THEOREM 6.12. If $C_{f F}^{k \uparrow}$ is unsatisfiable, then $C_{f F}$ is unsatisfiable modulo $\varphi_{(b, \oplus)}$.

Proof. We know from Thm. 5.7 and Thm. 6.2 that, modulo $\varphi_{(b, \oplus)}, C_{F}$ is equisatisfiable to $C_{f F}$. We prove that if $C_{f F}^{k \uparrow}$ is unsatisfiable, then $C_{F}$ is unsatisfiable. The contrapositive of this statement is that if $C_{F}$ is satisfiable, then $C_{f F}^{k \uparrow}$ is satisfiable. The constraints in the clauses of $C_{f F}^{k \uparrow}$ are stronger than the constraints in the corresponding clauses of $C_{F}$. Therefore, for all models $M$, if $M \vDash C_{F}$, then $M \vDash C_{f F}^{k \uparrow}$.

\section{SOLVING CHC MODULO ADT AND RDF}

In this section, we present RACER, an algorithm that solves CHCs of the form $C_{f F}$ (Eq. (7)) modulo a catamorphism axiom $\varphi_{(b, \oplus)}$ for $f$. RACER is sound: if it finds a solution, it is a solution for the original set of $\mathrm{CHCs}, C_{f}$, modulo the axiom; if it declares UNSAT, then the original set of $\mathrm{CHCs}$ 
modulo the axiom is UNSAT. Further, if $C_{f}$ is UNSAT, RACER is guaranteed to terminate. RACER works by simultaneously searching for $\mathcal{A}$-solutions of $C_{f F}^{k}$ as well as counter examples to $C_{f F}^{k \uparrow}$ while periodically increasing $k$. RACER is based on SPACER, an IC3-style algorithm for solving CHCs We first give background on SPACER and explain necessary notation (Sec. 7.1) and then explain RACER (Sec. 7.2).

\subsection{Background on SPACER}

SPACER is based on IC3, which was developed for safety Model Checking. For that reason, we switch terminology from logic to states and transitions (as explained in Sec. 3.3), instead of adapting IC3 to the terminology of the paper. Our implementation, however, works with general CHCs.

SPACER works by generating and blocking predecessors to Bad states called proof obligations (POB). Each time SPACER blocks a POB, it learns a lemma that blocks many similar POBs. Each time SPACER proves that a $\mathrm{POB}$ is reachable from the initial states, it computes reachable states to avoid generating the same POB again. For each predicate, the lemmas can be used to construct a may summary whereas the reachable states can be used to create a must summary. The may summaries are used to create $\mathcal{A}$-solutions.

Alg. 1 describes our contributions (highlighted) on top of the SpAcER algorithm. To make the presentation concise, we have used the following notation.

Notation. All formulas in the algorithm are over state variables $\bar{x}$. For a formula $\theta$ over state variables, we use the shorthand $\theta[\bar{y}]$ to mean $\theta[\bar{x} \mapsto \bar{y}]$. We assume that whenever a predicate $\mathcal{P}$ appears in the head of a Horn clause, it has the same variables as its arguments. We use the notation $\overline{\mathrm{UT}}^{\mathcal{P}}$ to represent the uninterpreted tail of predicate $\mathcal{P}$ : the sequence of all pairs $\langle Q, \bar{v}\rangle$ where $Q$ is an uninterpreted predicate and the literal $Q(\bar{v})$ appears in the tail of a Horn clause whose head is $\mathcal{P}$. We use the notation $\overline{\mathrm{UT}}_{i}^{\mathcal{P}}$ to mean the uninterpreted tail of the $i$ th Horn clause whose head is $\mathcal{P}$. As a special case, we use $\overline{\mathrm{UT}}^{\text {Bad }}$ to refer to the set of pairs of uninterpreted predicates and the argument variables in the tail of the Horn clause whose head is Bad. Let $\mathcal{Y}$ be a map from uninterpreted predicates to their summaries, where a summary is a formula over $\bar{x}$, and $\mathcal{Y}^{\mathcal{Q}}$ is the summary of uninterpreted predicate $Q$ in $\mathcal{Y}$. We use the shorthand $\mathcal{F}_{\mathcal{P}}(\boldsymbol{Y})$ to denote the predicate transformer of $\mathcal{P}$ defined as:

$$
\mathcal{F}_{\mathcal{P}}(\mathcal{Y})=\left(\text { Init }^{\mathcal{P}}\right)^{\prime} \vee \bigvee_{i}\left(\left(\bigwedge_{\langle Q, \bar{v}\rangle \in \overline{\mathrm{UT}}_{i}^{\mathcal{P}}} \boldsymbol{y}^{Q}[\bar{x} \mapsto \bar{v}]\right) \wedge T r_{i}\right)
$$

where $\operatorname{Init}^{\mathcal{P}}$ is the disjunction of tails of Horn clauses which have no uninterpreted predicates in their tails and whose head is $\mathcal{P}$ and $T r_{i}$ is the constraint in the $i$ th Horn clause whose head is $\mathcal{P}$. We use $R$ to denote the map from uninterpreted predicates to their must summaries. Similarly, we use $O_{i}$ to denote the map from uninterpreted predicates to their may summaries in the $i$ 'th frame. We denote the sequence $O_{0}, O_{1}, \ldots$ by $\bar{O}$, and write $\bar{O}_{i}$ to refer to $O_{i}$.A POB is represented as a triple $\langle\theta, i, \mathcal{P}\rangle$, where $\theta$ is formula in List $_{\mathrm{s}}+\mathrm{r}\left(\mathrm{List}_{\mathrm{s}}\right.$ is the sort of List and $\mathrm{r}$ is the return sort of $\mathrm{RDF}), i$ is a natural number representing the level at which $\theta$ is to be blocked and $\mathcal{P}$ denotes the uninterpreted predicate to which the POB belongs. For CHCs like those in Eq. (7), $\mathcal{P}$ can either be $I$, the uninterpreted predicate representing the desired inductive invariant of the program or $F$, the relationified RDF.

\subsection{The RACER Algorithm}

We are ready to explain the full algorithm. The input to RACER (Alg. 1) are CHCs of the form Eq. (7) together with a function axiom for $f$ and the output is SAFE/UNSAFE. RACER need not terminate. 
Algorithm 1: The RACER algorithm. The parts of the algorithm which are highlighted in green (resp. yellow) are the only places that use $\alpha_{r f}^{k}$ (resp. $\alpha_{r f}^{k \uparrow}$ ) abstraction. Pink highlights the increment of $k$. The rest of the algorithm is the same as SPACER.
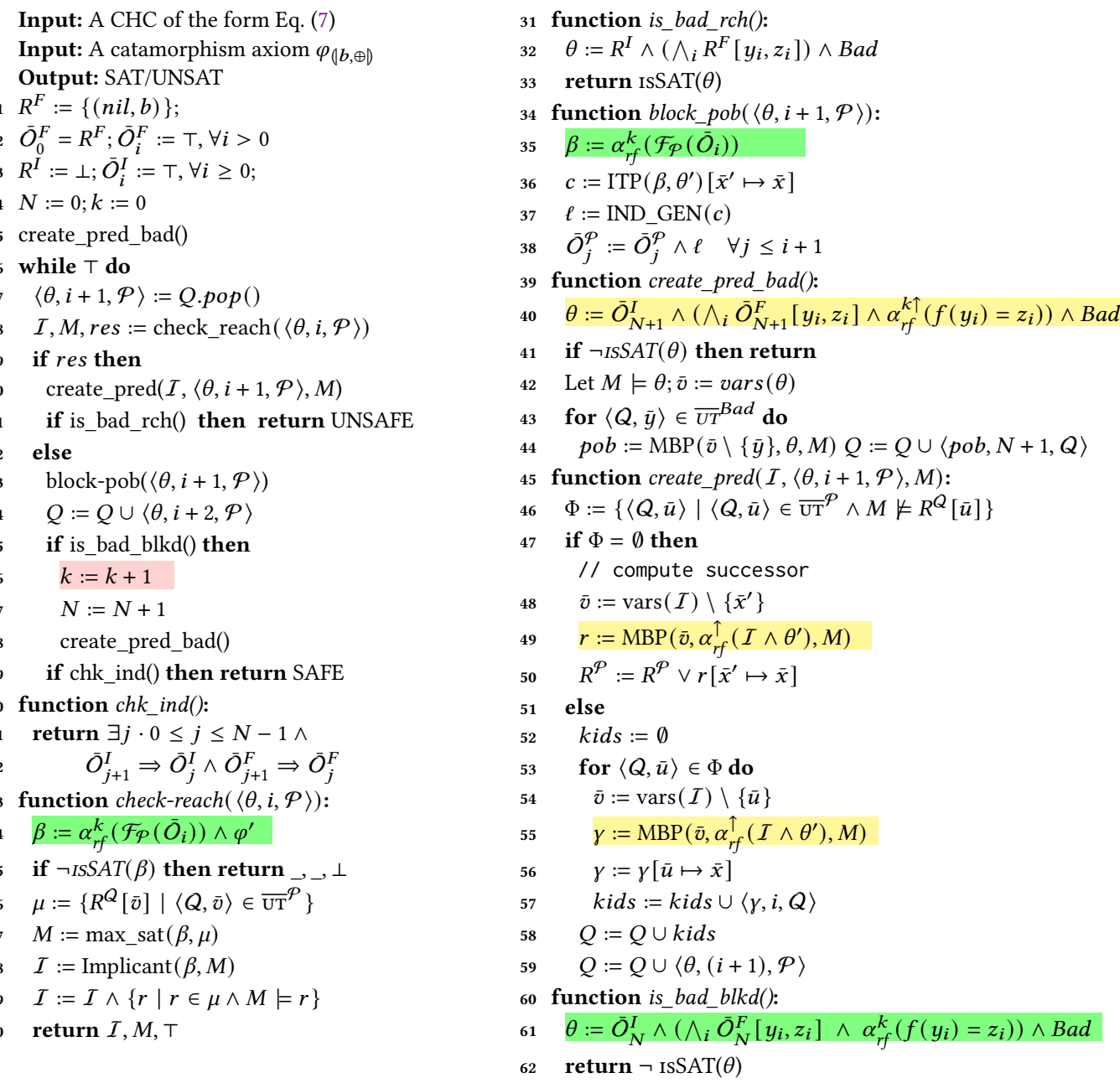

The Initialization phase. RACER initializes both $R^{F}$ and $\bar{O}_{0}^{F}$ to $\{(n i l, b)\}$ (Line 1). Note that we are using a set of states to represent a formula over variables $\bar{x}$. Next, $R^{I}$ is initialized with $\perp$ and all the $\bar{O}^{I}$ with T. These initialization steps are sound because in Eq. (7), $F$ is the only predicate that appears in the head of a clause that has no predicate in the body. After initialization, RACER creates predecessors to Bad and add them to the queue (line 5).

IC3-part of the algorithm. In each iteration RACER calls the check_reach function to check the reachability of a POB at a particular level (line 8). The check_reach function first checks whether the $\mathrm{POB}$ is blocked using may summaries (line 25). If the check is satisfiable, the function finds a model that satisfies as many must summaries as possible using a max_sat algorithm. The check_reach 
function returns $T$ only if the may summaries are not strong enough to block the POB at this level. In this case, the algorithm calls the create_pred function to create and add predecessor POBs to the queue (line 10). Otherwise, it calls the block_pob to learn a lemma that blocks the POB (line 13). The create_pred function (line 45) uses Model Based Projection (MBP) [Komuravelli et al. 2016] to create a predecessor $\mathrm{POB}$ for all the predicates in the tail whose must summaries are not satisfied by the model (but their may summaries are). If there are no such predicates, the POB is reachable from the must summaries. In this case, the algorithm computes a reachable state that intersects with the POB using MBP (line 49) and adds it to the set of reachable states. RACER routinely checks whether $\mathrm{Bad}$ is reachable (line 11) and increases the depth of exploration when Bad is blocked (line 15).

The role of abstractions. When checking for one-step reachability, RACER uses the $k$-instantiation abstraction to abstract the predicate transformer (line 24). RACER employs the same abstraction when learning a lemma (line 34) and when checking whether Bad is blocked (line 60). This ensures any inductive invariant RACER finds is an $\mathcal{A}$-solution to $C_{f F}^{k}$. RACER employs both uninterpreted function abstraction and $k$-instantiation abstraction when computing predecessors (line 55) and successors (line 49). This ensures that if Bad is reachable, it is reachable in $C_{f F}^{k \uparrow}$.

MBP. RACER generates predecessor POBs by using Model Based Projection (MBP) [Komuravelli et al. 2016] to eliminate post state variables. Given a quantifier free formula $\theta$, a model $M \vDash \theta$, and a set of constants $\bar{x}, M B P(\theta, \bar{x}, M)$ is a model preserving under-approximation of $\theta$ projected onto $\Sigma \backslash \bar{x}$. That is, $\operatorname{MBP}(\theta, \bar{x}, M)$ is a quantifier free formula $\psi$ such that (1) $\psi$ does not contain the constants $\bar{x},(2)$ constants of $\psi$ are a subset of constants of $\theta$, and (3) $\psi$ under-approximates the result of eliminating $\bar{x}$ from $\theta: \psi \Rightarrow(\exists \bar{x} \cdot \theta)$. While there are algorithms to compute MBP for the theory of ADTs [Bjørner and Janota 2015], it is not clear how to compute MBP in the presence of UF because of lack of quantifier elimination. The abstractions ensure that there are no UF when MBP is applied. Thus, RACER uses MBP for ADT+LIA from [Bjørner and Janota 2015].

Progress. From the properties of IC3, it follows that, whenever RACER or SPACER increments the depth of exploration ( $N$ in Alg. 1), there are no counterexamples of lower depth. We say that RACER (SPACER) makes progress if all non-terminating executions of RACER (SPACER) increment the depth of exploration infinitely often. In RACER (Alg. 1), this amounts to executing line 15 infinitely often on all non-terminating executions.

SPACER makes progress as long as the underlying theory is decidable and has a finite MBP. RACER keeps the underlying theory decidable by using abstractions to ensure that all queries are quantifier free formulas without RDFs. However, unlike SPACER, RACER does not use the same formula to check reachability of a POB and to compute its predecessors. Instead, RACER uses only the $k$-instantiation abstraction when checking reachability and uses both $k$-instantiation abstraction as well as uninterpreted function abstraction when computing predecessors using MBP. This makes arguing about progress a little tricky.

We first make two assumptions and show that RACER makes progress under those assumptions. We then relax those assumptions and argue why RACER still makes progress. Assume that $k$ is fixed and that, during the one-step reachability check RACER employs both uninterpreted function abstraction as well as the $k$-instantiation abstraction instead of doing only the $k$-instantiation abstraction. Therefore, just like in the original SPACER, the formula to check reachability of a $\mathrm{POB}$ and the formula to compute its predecessors are the same. Since the MBP for the underlying theory is finite [Bjørner and Janota 2015], each POB can only have a finite number of predecessors. Therefore, RACER makes progress under these assumptions. Now we relax the second assumption and employ only the $k$-instantiation abstraction during one-step reachability check. Let $\alpha_{r f}^{k}(\theta)$ be 
the $k$-instantiation abstracted formula that RACER uses to check reachability. We know that $\alpha_{r f}^{k \uparrow}(\theta)$ is weaker than $\alpha_{r f}^{k}(\theta)$. Therefore, all models of $\alpha_{r f}^{k}(\theta)$ are also models of $\alpha_{r f}^{k \uparrow}(\theta)$. Hence, when RACER computes MBP, no new models have been introduced just because we relaxed this assumption. Therefore, MBP is still be finite and RACER still makes progress. As for the first assumption, RACER makes progress as long as $k$ is not increased too frequently. In our implementation, $k$ is increased by 1 each time $N$ is increased. Since the depth of counterexamples in any unsatisfiable CHCs is finite, the progress guarantee ensures that RACER is guaranteed to terminate on all unsatisfiable CHCs. Combining this with the results from earlier sections, we conclude the following:

THEOREM 7.1. Let $C_{f F}$ be a set of reltionified CHCs modulo function axiom $\varphi_{(b, \oplus)}$. If $C_{f F}$ is UNSAT, RACER terminates and returns UNSAT. If $C_{f F}$ is satisfiable, if RACER terminates, it returns a solution to $C_{f F}$.

\section{IMPLEMENTATION AND EVALUATION}

We have implemented RACER on top of SPACER in $\mathrm{Z3}^{3}$. Our implementation supports CHCs modulo multiple ADTs as well as multiple RDFs as long as all RDFs are relationified (i.e., in the form Eq. (7)).

Benchmark description. While we are mainly interested in solving CHCs encoding imperative programs, to show the robustness of our approach, we evaluate RACER on CHC benchmarks obtained from two sources. The first group, called RusT-BENCH, contains $87 \mathrm{CHCs}$ encoding safety of imperative Rust programs from [Matsushita et al. 2020]. The second group, called LEON-BENCH, contains $64 \mathrm{CHCs}$ encoding safety of ADT manipulating functional programs [De Angelis et al. 2020].

Getting benchmarks into the desired form. While the benchmarks encode programs manipulating ADTs, they do not take advantage of RDFs. That is, the CHCs in these benchmarks capture programs using only uninterpreted predicates. They are relationified $\mathrm{CHCs}$ conjoined with positive relation constraints of RDFs (of the form Eq. (4), extended to include multiple $F$ predicates).

We convert these $\mathrm{CHCs}$ into the desired form ${ }^{4}$ of $C_{f F}$ (Eq. (7)) modulo all function axioms by identifying some predicates $F$ with the RDFs $f$ they correspond to, and recovering their RDF definitions. Specifically, we identify a subset of program methods as implementing RDFs and all other program methods (including recursive ones) as an implementation of some imperative program. For all program methods that define RDFs, we produce both their $\mathrm{CHC}$ encoding (positive relation constraint) and RDF axioms. All program methods that compute arithmetic properties of ADTs are considered as RDFs. For example, a method that checks whether a given list contains an element is considered an RDF, whereas a method that appends a given element to a list is not.

We use term abstraction to facilitate solutions that do not contain RDF applications. Specifically, we introduce new (arithmetic) variables to represent applications of RDFs that are necessary to prove safety. These variables are added to predicates in the CHCs as arguments that can be used in solutions. The equalities between the variables and the RDF applications that they represent are added to the bodies of the CHCs. Term abstraction is done greedily: for each RDF and each predicate argument, if the RDF can be applied either to the argument itself or, if the argument is a record type, to a selector applied to the argument, a new variable is introduced to capture the $\mathrm{RDF}$ application. We stress that this process is completely automatic. That is, we use the same exact input for all the tools in our evaluation. For our tool, we do a simple preprocessing step that identifies relationfied RDFs, re-encodes them as RDFs, and generates terms for term abstraction.

\footnotetext{
${ }^{3}$ Our implementation is available at https://github.com/hgvk94/z3/tree/racer

${ }^{4}$ Both modified and unmodified benchmarks are publicly available at https://doi.org/10.5281/zenodo.5083780.
} 
Table 1. Comparing solved instances on RuST-BENCH. In each cell, the first entry is the number of SAT instances solved and the second entry is the number of UNSAT instances solved in that category.

\begin{tabular}{llllll}
\hline Category & Count & Eldarica & SPACER & HOICE & RACER \\
\hline Programs with RDFs & 44 & $0 / 0$ & $1 / 8$ & $18 / 3$ & $36 / 0$ \\
Programs without RDFs & 43 & $7 / 7$ & $22 / 20$ & $13 / 15$ & $22 / 20$ \\
\hline Total & 87 & $7 / 7$ & $23 / 28$ & $31 / 18$ & $58 / 20$ \\
\hline
\end{tabular}

Evaluation on RUST-BENCH. The Rust-BENCH benchmark suite encodes Rust programs that manipulate ADTs. Of the 87 benchmarks, only 44 manipulate recursive ADTs (lists and trees) and contain (recovered) RDFs. The RDFs computed the length of lists, size of trees and sum of elements of trees and lists. On these benchmarks, we compare RACER with Eldarica ${ }^{5}$ [Hojjat and Rümmer 2018], SpACER, and HoICE - state-of-the-art tools that support CHCs modulo ADT. Since none of these solvers support $\mathrm{CHCs}$ modulo RDFs, they cannot be run on our modified benchmarks. To make a comparison, we run them on the original set of benchmarks from [Matsushita et al. 2020]. We ran all four solvers with a timeout of 100s. Our results are shown in Tab. 1. In total, RAcER solves 29 (resp. 27) more benchmarks than HoIce (resp. SPACER). When we only consider programs that contain RDFs, RACER solves 15 (resp. 27) more instances than HoIce (resp. SpACER). The superiority of RACER is clear when looking at the number of SAT instances it solves (58) compared to HoIcE (31) and SPACER (23).

Evaluation on LEON-BENCH. The benchmarks in LEON-BENCH encode verification condition validation queries generated by Leon [Suter et al. 2011]. The benchmarks encode operations on three types of ADTs: Queues, Trees, and Heaps. The RDFs compute size of the ADTs, checks membership in them, removes the last element, and checks whether they are sorted. We present our evaluation results on SAT and UNSAT CHCs separately. We further split the SAT benchmarks based on the type of ADTs.

On LEON-BENCH, we compare RACER with three CHC solvers: VeriMap [De Angelis et al. 2020], SpACER, Eladarica and with CVC4 [Reynolds and Kuncak 2015] (with the subgoal generation heuristic enabled). For our evaluation, we use three different versions of these benchmarks: (1) to evaluate Verimap, Eldarica, and SPACER, we use the CHC encoding over ADTs and LIA, (2) to evaluate $\mathrm{CVC}$, we use the original verification condition validation queries but without any of the subgoals, as done in [De Angelis et al. 2020], and (3) to evaluate RACER, we conjoin RDFs to CHCs as mentioned earlier.

Tab. 2 shows the results of our evaluation. In summary, RACER is quite competitive with all other solvers even though RACER was designed for verifying imperative programs and not functional programs. In fact, in almost all categories, RACER solves benchmarks that other solvers do not solve.

We could not compare RACER with all solvers on all benchmarks because the tools do not support exactly the same inputs: Eldarica, HoICE, and SpACER support CHCs in the SMT2-LIB format, VeriMap supports CHCs modulo ADTs but in the Prolog format, and the inductive reasoning in CVC4 works with SMT-LIB formulas. We also repeated the experiments with a larger timeout of 30 minutes and did not see any change in number of instances solved for any of the solvers.

\footnotetext{
${ }^{5}$ We could not make use of Eldarica's support for size constraints because in our benchmarks, RDFs are not restricted to size constraints.
} 
Table 2. Comparing RACER with SPACER, Eldarica, CVC4, and VeriMAP on LeON-Bench. The first three rows show SAT instances. The UNSAT row shows UNSAT instances from all three categories. The number of unique instances solved are shown in brackets.

\begin{tabular}{lllllll}
\hline Category & Count & Eldarica & VeriMAP & CVC4 & SPACER & RACER \\
\hline Queue & 11 & 2 & $4(2)$ & 3 & 0 & $4(1)$ \\
Tree & 18 & 0 & $12(1)$ & $7(1)$ & 0 & 7 \\
Heap & 12 & 0 & $7(3)$ & 1 & 0 & $4(1)$ \\
\hline UNSAT & 23 & 20 & 13 & 0 & $23(1)$ & 22 \\
\hline Total & 64 & 22 & 36 & 11 & 23 & 37 \\
\hline
\end{tabular}

\section{RELATED WORK}

The theory of ADTs and RDFs is part of the SMT-LIB v2.6 standard [Barrett et al. 2017] and is well supported by the major SMT solvers. Most decision procedures for ADTs are based on finite instantiation of ADT axioms [Barrett et al. 2007; Bjørner 1999; Oppen 1980]. The combination of ADTs and RDFs is undecidable. Most existing solvers use finite unrollings of RDF definitions to check satisfiability [Suter et al. 2010]. This is similar to how quantifiers are typically handled. The solver returns UNSAT if some finite unrolling of RDFs is sufficient for unsatisfiability, otherwise, the solver might return unknown. This is usually not a significant hurdle for deductive-style verification tools that treat unknown as unproven and rely on the user to supply additional lemmas to help the solver. At the same time, this is a serious issue for all automated verification tools since they rely on the solver's SAT answers to drive abstraction refinement. We overcome this problem by unrolling RDFs and then either abstracting the result with uninterpreted functions or abstracting RDFs altogether. In both cases, the abstract formulas are decidable and we use their models to drive the abstraction-refinement process within RACER.

In some cases, extending ADTs with RDFs remains decidable. A particularly interesting class of decidable RDFs is called (generalized) infinitely surjective catamorphisms [Pham et al. 2016; Suter et al. 2010, 2011]. The class includes many useful RDFs over ADTs including length of a list, height and size of a tree, as well as various filters over lists and trees. However, showing that a given RDF is an infinitely surjective catamorphism is not easy, which limits their application in a completely automated procedure such as RACER. Interestingly, in our running example (Fig. 1), RACER is able to automatically generate the necessary property of the length function to show that it is infinitely surjective, and, with this lemma, SMT queries involving length become decidable. That is, the existing procedure of finite unfolding terminates with either SAT or UNSAT.

More specialized restrictions, such as restricting to specific catamorphisms have been considered as well [Hojjat and Rümmer 2017; Zhang et al. 2006]. In contrast, we do not restrict the definition of the catamorphism, and automatically infer supporting lemmas for surjectivity. Moreover, our approach does not differentiate between catamorphisms and arbitrary RDFs. Of course, our procedure in incomplete and need not terminate.

Prior to inclusion of RDFs in the SMT-LIB standard and their native support in solvers, deductive verification tools have shown that dynamic unfolding of RDFs can be simulated by quantifier instantiation. Most prominent is the so called fuel approach of Amin et al. [Amin et al. 2014] that uses ADTs to encode ordinals to control number of unrollings of RDFs. Current state-of-the-art procedures for RDFs work directly within an SMT solver and unroll RDFs by adding (and removing) constraints to the SMT solver using its incremental interface. This is also what we do in our 
implementation. One of the advantages is that this enables combining RDFs with other theories and without enabling quantifier support in the solver. We do not compare with [Amin et al. 2014], or any deductive-style verification approach, since they require the user to provide candidate inductive invariants while RACER is completely automatic.

Automatic inductive reasoning is studied in the context of SMT (e.g., [Reynolds and Kuncak 2015]), automated theorem proving (e.g., [Reger and Voronkov 2019]), and deductive program analysis (e.g., [Leino 2012]). However, these works do not apply directly to CHC-solving, and especially in combination with other theories such as LIA. For example, the TIP benchmarks [Rosén and Smallbone 2015] focus on functional programs and model integers with natural numbers.

There are a number of CHC Solvers that support ADT constraints. VeriMAP [De Angelis et al. 2018] transforms CHCs with ADTs to CHCs over basic types, by using folding/unfolding and by introducing difference predicates [De Angelis et al. 2020]. In this way, VeriMAP separates ADT reasoning from $\mathrm{CHC}$ solving. VeriMAP can determine satisfiability of $\mathrm{CHCs}$ but cannot generate solutions. Most importantly, VeriMAP is unsound for UNSAT answers. In contrast, our approach is sound for both SAT and UNSAT and generates solutions that can be independently verified. CHC solver HoIce [Champion et al. 2018] can produce solutions containing RDFs (but it does not allow RDFs in the input). It is based on the ICE framework that iteratively enumerates the space of solutions including a restricted set of RDFs. In contrast to RACER, HoICE does not provide progress guarantees nor always generates a counterexample. The RInGen [Kostyukov et al. 2021] solver infers solutions to CHCs modulo ADTs by treating all symbols as uninterpreted (thus, it does not support arithmetic). It uses a finite model finder to infer a finite model for the resulting FoL formula and extends it to a solution expressed using a tree automaton. Since RInGen treats all symbols as uninterpreted, it does not support types and could not be compared to our approach.

In SMT, it is common to abstract complex functions (e.g., multiplication, RDFs) by uninterpreted functions. However, lifting this abstraction effectively to $\mathrm{CHC}$ is still an open challenge. One issue is that in the context of CHCs, uninterpreted functions are universally quantified. That is, the solver must find a solution that works for all interpretaions of UFs, not synthesize a model for these functions. An interested reader is referred to [Bjørner et al. 2015] for the formal definition. We avoid dealing with UFs by requiring that all functions are explicitly defined and UFs are only used as local abstraction during the algorithm. This is similar to support for UFs in Euforia [Bueno and Sakallah 2019], except that we deal with recursively defined functions rather than bit-vector terms.

In this paper, we present a procedure for solving CHCs modulo ADTs and RDFs. Our approach sidesteps the undecidability of the underlying logic by simultaneously approximating the RDFs using multiple abstractions. One abstraction compiles RDF to $\mathrm{CHCs}$ while preserving satisfiability (but not solutions). It is used to detect unsatisfiability of CHCs (i.e., counterexamples to safety verification). The other abstraction, parameterized by the depth of unfolding $k$, replaces RDFs by their finite unfolding. As $k$ increases, the abstraction enables more solutions that are potentially lost by the first abstraction. The two abstractions are explored in tandem in an IC3-style algorithm. Remarkably, the algorithm is able to automatically combine learning inductive invariants over ADTs and RDFs with learning inductive lemmas of RDFs.

While our presentation focuses on specific RDFs - catamorphisms - our results are more general. They apply to arbitrary well defined RDFs. The only requirements are that the function is total and it is possible to isolate the base- and recursive-cases in the definition.

We have implemented our technique in the SPACER CHC-solver of Z3. Our evaluation shows significant improvement of our technique over state-of-the-art $\mathrm{CHC}$ solvers on imperative programs while still being competitive on functional programs.

In this work, we assumed that RDFs are given (or mined) in the original problem. It is interesting to extend the work further by synthesizing RDFs together with an inductive invariant. We believe 
that many simple catamorphisms, such as length, size, height, etc., and their variants, can be constructed automatically during the search. We leave exploring this challenging direction to future work.

\section{ACKNOWLEDGMENTS}

The research leading to these results has received funding from the European Research Council under the European Union's Horizon 2020 research and innovation programme (grant agreement No [759102-SVIS]). This research was partially supported by the United States-Israel Binational Science Foundation (BSF) grant No. 2016260, and the Israeli Science Foundation (ISF) grant No. 1810/18. We acknowledge the support of the Natural Sciences and Engineering Research Council of Canada (NSERC) and MathWorks Inc.

\section{REFERENCES}

Francesco Alberti, Roberto Bruttomesso, Silvio Ghilardi, Silvio Ranise, and Natasha Sharygina. 2012. Lazy Abstraction with Interpolants for Arrays. In Logic for Programming, Artificial Intelligence, and Reasoning - 18th International Conference, LPAR-18, Mérida, Venezuela, March 11-15, 2012. Proceedings (Lecture Notes in Computer Science), Nikolaj Bjørner and Andrei Voronkov (Eds.), Vol. 7180. Springer, 46-61. https://doi.org/10.1007/978-3-642-28717-6_7

Nada Amin, K. Rustan M. Leino, and Tiark Rompf. 2014. Computing with an SMT Solver. In Tests and Proofs - 8th International Conference, TAP@STAF 2014, York, UK, July 24-25, 2014. Proceedings (Lecture Notes in Computer Science), Martina Seidl and Nikolai Tillmann (Eds.), Vol. 8570. Springer, 20-35. https://doi.org/10.1007/978-3-319-09099-3_2

Clark Barrett, Pascal Fontaine, and Cesare Tinelli. 2017. The SMT-LIB Standard: Version 2.6. Technical Report. Department of Computer Science, The University of Iowa. Available at www. SMT-LIB. org.

Clark W. Barrett, Igor Shikanian, and Cesare Tinelli. 2007. An Abstract Decision Procedure for a Theory of Inductive Data Types. F. Satisf. Boolean Model. Comput. 3, 1-2 (2007), 21-46. https://doi.org/10.3233/sat190028

Nikolaj Bjørner. 1999. Integrating Decision Procedures for Temporal Verification. Ph.D. Dissertation. Stanford University, Stanford, CA, USA. Advisor(s) Manna, Zohar. AAI9924398.

Nikolaj Bjørner, Arie Gurfinkel, Kenneth L. McMillan, and Andrey Rybalchenko. 2015. Horn Clause Solvers for Program Verification. In Fields of Logic and Computation II - Essays Dedicated to Yuri Gurevich on the Occasion of His 75th Birthday (Lecture Notes in Computer Science), Lev D. Beklemishev, Andreas Blass, Nachum Dershowitz, Bernd Finkbeiner, and Wolfram Schulte (Eds.), Vol. 9300. Springer, 24-51. https://doi.org/10.1007/978-3-319-23534-9_2

Nikolaj Bjørner and Mikolás Janota. 2015. Playing with Quantified Satisfaction. In 20th International Conferences on Logic for Programming, Artificial Intelligence and Reasoning - Short Presentations, LPAR 2015, Suva, Fiji, November 24-28, 2015 (EPiC Series in Computing), Ansgar Fehnker, Annabelle McIver, Geoff Sutcliffe, and Andrei Voronkov (Eds.), Vol. 35. EasyChair, 15-27.

Denis Bueno and Karem A. Sakallah. 2019. EUForia: Complete Software Model Checking with Uninterpreted Functions. In Verification, Model Checking, and Abstract Interpretation - 20th International Conference, VMCAI 2019, Cascais, Portugal, fanuary 13-15, 2019, Proceedings (Lecture Notes in Computer Science), Constantin Enea and Ruzica Piskac (Eds.), Vol. 11388. Springer, 363-385. https://doi.org/10.1007/978-3-030-11245-5_17

Adrien Champion, Tomoya Chiba, Naoki Kobayashi, and Ryosuke Sato. 2018. ICE-Based Refinement Type Discovery for Higher-Order Functional Programs. In Tools and Algorithms for the Construction and Analysis of Systems - 24th International Conference, TACAS 2018, Held as Part of the European foint Conferences on Theory and Practice of Software, ETAPS 2018, Thessaloniki, Greece, April 14-20, 2018, Proceedings, Part I (Lecture Notes in Computer Science), Dirk Beyer and Marieke Huisman (Eds.), Vol. 10805. Springer, 365-384. https://doi.org/10.1007/978-3-319-89960-2_20

CHC-COMP. 2021. CHC-COMP. https://chc-comp.github.io.

Emanuele De Angelis, Fabio Fioravanti, Alberto Pettorossi, and Maurizio Proietti. 2018. Solving Horn Clauses on Inductive Data Types Without Induction. Theory Pract. Log. Program. 18, 3-4 (2018), 452-469. https://doi.org/10.1017/ S1471068418000157

Emanuele De Angelis, Fabio Fioravanti, Alberto Pettorossi, and Maurizio Proietti. 2020. Removing Algebraic Data Types from Constrained Horn Clauses Using Difference Predicates. In Automated Reasoning - 10th International foint Conference, IfCAR 2020, Paris, France, July 1-4, 2020, Proceedings, Part I (Lecture Notes in Computer Science), Nicolas Peltier and Viorica Sofronie-Stokkermans (Eds.), Vol. 12166. Springer, 83-102. https://doi.org/10.1007/978-3-030-51074-9_6

Grigory Fedyukovich, Samuel J. Kaufman, and Rastislav Bodík. 2017. Sampling invariants from frequency distributions. In 2017 Formal Methods in Computer Aided Design, FMCAD 2017, Vienna, Austria, October 2-6, 2017, Daryl Stewart and Georg Weissenbacher (Eds.). IEEE, 100-107. https://doi.org/10.23919/FMCAD.2017.8102247 
Grigory Fedyukovich, Samuel J. Kaufman, and Rastislav Bodík. 2020. Learning inductive invariants by sampling from frequency distributions. Formal Methods Syst. Des. 56, 1 (2020), 154-177. https://doi.org/10.1007/s10703-020-00349-x

Jean-Christophe Filliâtre and Andrei Paskevich. 2013. Why3 - Where Programs Meet Provers. In Programming Languages and Systems - 22nd European Symposium on Programming, ESOP 2013, Held as Part of the European foint Conferences on Theory and Practice of Software, ETAPS 2013, Rome, Italy, March 16-24, 2013. Proceedings (Lecture Notes in Computer Science), Matthias Felleisen and Philippa Gardner (Eds.), Vol. 7792. Springer, 125-128. https://doi.org/10.1007/978-3-642-37036-6_8

Cormac Flanagan, Rajeev Joshi, and K. Rustan M. Leino. 2001. Annotation inference for modular checkers. Inf. Process. Lett. 77, 2-4 (2001), 97-108. https://doi.org/10.1016/S0020-0190(00)00196-4

Cormac Flanagan and K. Rustan M. Leino. 2001. Houdini, an Annotation Assistant for ESC/Java. In FME 2001: Formal Methods for Increasing Software Productivity, International Symposium of Formal Methods Europe, Berlin, Germany, March 12-16, 2001, Proceedings (Lecture Notes in Computer Science), José Nuno Oliveira and Pamela Zave (Eds.), Vol. 2021. Springer, 500-517. https://doi.org/10.1007/3-540-45251-6_29

Pranav Garg, Daniel Neider, P. Madhusudan, and Dan Roth. 2016. Learning invariants using decision trees and implication counterexamples. In Proceedings of the 43rd Annual ACM SIGPLAN-SIGACT Symposium on Principles of Programming Languages, POPL 2016, St. Petersburg, FL, USA, January 20 - 22, 2016, Rastislav Bodík and Rupak Majumdar (Eds.). ACM, 499-512. https://doi.org/10.1145/2837614.2837664

Sergey Grebenshchikov, Nuno P. Lopes, Corneliu Popeea, and Andrey Rybalchenko. 2012. Synthesizing software verifiers from proof rules. In ACM SIGPLAN Conference on Programming Language Design and Implementation, PLDI '12, Beijing, China - June 11 - 16, 2012, Jan Vitek, Haibo Lin, and Frank Tip (Eds.). ACM, 405-416. https://doi.org/10.1145/2254064. 2254112

Krystof Hoder and Nikolaj Bjørner. 2012. Generalized Property Directed Reachability. In Theory and Applications of Satisfiability Testing - SAT 2012 - 15th International Conference, Trento, Italy, June 17-20, 2012. Proceedings (Lecture Notes in Computer Science), Alessandro Cimatti and Roberto Sebastiani (Eds.), Vol. 7317. Springer, 157-171. https: //doi.org/10.1007/978-3-642-31612-8_13

Hossein Hojjat and Philipp Rümmer. 2017. Deciding and Interpolating Algebraic Data Types by Reduction. In 19th International Symposium on Symbolic and Numeric Algorithms for Scientific Computing, SYNASC 2017, Timisoara, Romania, September 21-24, 2017, Tudor Jebelean, Viorel Negru, Dana Petcu, Daniela Zaharie, Tetsuo Ida, and Stephen M. Watt (Eds.). IEEE Computer Society, 145-152. https://doi.org/10.1109/SYNASC.2017.00033

Hossein Hojjat and Philipp Rümmer. 2018. The ELDARICA Horn Solver. In 2018 Formal Methods in Computer Aided Design, FMCAD 2018, Austin, TX, USA, October 30 - November 2, 2018, Nikolaj Bjørner and Arie Gurfinkel (Eds.). IEEE, 1-7. https://doi.org/10.23919/FMCAD.2018.8603013

Anvesh Komuravelli, Arie Gurfinkel, and Sagar Chaki. 2016. SMT-based model checking for recursive programs. Formal Methods Syst. Des. 48, 3 (2016), 175-205. https://doi.org/10.1007/s10703-016-0249-4

Yurii Kostyukov, Dmitry Mordvinov, and Grigory Fedyukovich. 2021. Beyond the elementary representations of program invariants over algebraic data types. In PLDI '21: 42nd ACM SIGPLAN International Conference on Programming Language Design and Implementation, Virtual Event, Canada, fune 20-25, 20211, Stephen N. Freund and Eran Yahav (Eds.). ACM, 451-465. https://doi.org/10.1145/3453483.3454055

K. Rustan M. Leino. 2010. Dafny: An Automatic Program Verifier for Functional Correctness. In Logic for Programming, Artificial Intelligence, and Reasoning - 16th International Conference, LPAR-16, Dakar, Senegal, April 25-May 1, 2010, Revised Selected Papers (Lecture Notes in Computer Science), Edmund M. Clarke and Andrei Voronkov (Eds.), Vol. 6355. Springer, 348-370. https://doi.org/10.1007/978-3-642-17511-4_20

K. Rustan M. Leino. 2012. Automating Induction with an SMT Solver. In Verification, Model Checking, and Abstract Interpretation - 13th International Conference, VMCAI 2012, Philadelphia, PA, USA, fanuary 22-24, 2012. Proceedings (Lecture Notes in Computer Science), Viktor Kuncak and Andrey Rybalchenko (Eds.), Vol. 7148. Springer, 315-331. https://doi.org/10.1007/978-3-642-27940-9_21

Christof Löding, P. Madhusudan, and Lucas Peña. 2018. Foundations for natural proofs and quantifier instantiation. Proc. ACM Program. Lang. 2, POPL (2018), 10:1-10:30. https://doi.org/10.1145/3158098

Yusuke Matsushita, Takeshi Tsukada, and Naoki Kobayashi. 2020. RustHorn: CHC-Based Verification for Rust Programs. In Programming Languages and Systems - 29th European Symposium on Programming, ESOP 2020, Held as Part of the European Joint Conferences on Theory and Practice of Software, ETAPS 2020, Dublin, Ireland, April 25-30, 2020, Proceedings (Lecture Notes in Computer Science), Peter Müller (Ed.), Vol. 12075. Springer, 484-514. https://doi.org/10.1007/978-3-030-44914-8_18

Peter Müller, Malte Schwerhoff, and Alexander J. Summers. 2016. Viper: A Verification Infrastructure for Permission-Based Reasoning. In Verification, Model Checking, and Abstract Interpretation - 17th International Conference, VMCAI 2016, St. Petersburg, FL, USA, January 17-19, 2016. Proceedings (Lecture Notes in Computer Science), Barbara Jobstmann and K. Rustan M. Leino (Eds.), Vol. 9583. Springer, 41-62. https://doi.org/10.1007/978-3-662-49122-5_2

Derek C. Oppen. 1980. Reasoning About Recursively Defined Data Structures. F. ACM 27, 3 (1980), 403-411. https: //doi.org/10.1145/322203.322204 
Tuan-Hung Pham, Andrew Gacek, and Michael W. Whalen. 2016. Reasoning About Algebraic Data Types with Abstractions. f. Autom. Reason. 57, 4 (2016), 281-318. https://doi.org/10.1007/s10817-016-9368-2

Giles Reger and Andrei Voronkov. 2019. Induction in Saturation-Based Proof Search. In Automated Deduction - CADE 27 27th International Conference on Automated Deduction, Natal, Brazil, August 27-30, 2019, Proceedings (Lecture Notes in Computer Science), Pascal Fontaine (Ed.), Vol. 11716. Springer, 477-494. https://doi.org/10.1007/978-3-030-29436-6_28

Andrew Reynolds and Viktor Kuncak. 2015. Induction for SMT Solvers. In Verification, Model Checking, and Abstract Interpretation - 16th International Conference, VMCAI 2015, Mumbai, India, fanuary 12-14, 2015. Proceedings (Lecture Notes in Computer Science), Deepak D’Souza, Akash Lal, and Kim Guldstrand Larsen (Eds.), Vol. 8931. Springer, 80-98. https://doi.org/10.1007/978-3-662-46081-8_5

Dan Rosén and Nicholas Smallbone. 2015. TIP: Tools for Inductive Provers. In Logic for Programming, Artificial Intelligence, and Reasoning - 20th International Conference, LPAR-20 2015, Suva, Fiji, November 24-28, 2015, Proceedings (Lecture Notes in Computer Science), Martin Davis, Ansgar Fehnker, Annabelle McIver, and Andrei Voronkov (Eds.), Vol. 9450. Springer, 219-232. https://doi.org/10.1007/978-3-662-48899-7_16

Philippe Suter, Mirco Dotta, and Viktor Kuncak. 2010. Decision procedures for algebraic data types with abstractions. In Proceedings of the 37th ACM SIGPLAN-SIGACT Symposium on Principles of Programming Languages, POPL 2010, Madrid, Spain, January 17-23, 2010, Manuel V. Hermenegildo and Jens Palsberg (Eds.). ACM, 199-210. https://doi.org/10.1145/ 1706299.1706325

Philippe Suter, Ali Sinan Köksal, and Viktor Kuncak. 2011. Satisfiability Modulo Recursive Programs. In Static Analysis - 18th International Symposium, SAS 2011, Venice, Italy, September 14-16, 2011. Proceedings (Lecture Notes in Computer Science), Eran Yahav (Ed.), Vol. 6887. Springer, 298-315. https://doi.org/10.1007/978-3-642-23702-7_23

Ting Zhang, Henny B. Sipma, and Zohar Manna. 2004. Decision Procedures for Recursive Data Structures with Integer Constraints. In Automated Reasoning - Second International foint Conference, IfCAR 2004, Cork, Ireland, fuly 4-8, 2004, Proceedings (Lecture Notes in Computer Science), David A. Basin and Michaël Rusinowitch (Eds.), Vol. 3097. Springer, 152-167. https://doi.org/10.1007/978-3-540-25984-8_9

Ting Zhang, Henny B. Sipma, and Zohar Manna. 2006. Decision procedures for term algebras with integer constraints. Inf. Comput. 204, 10 (2006), 1526-1574. https://doi.org/10.1016/j.ic.2006.03.004

He Zhu, Stephen Magill, and Suresh Jagannathan. 2018. A data-driven CHC solver. In Proceedings of the 39th ACM SIGPLAN Conference on Programming Language Design and Implementation, PLDI 2018, Philadelphia, PA, USA, fune 18-22, 2018, Jeffrey S. Foster and Dan Grossman (Eds.). ACM, 707-721. https://doi.org/10.1145/3192366.3192416 